\title{
Microscopic Computer Simulation of Fluids
}

\author{
Patricio Cordero ${ }^{1}$ and Dino Risso ${ }^{2}$ \\ 1 Departamento de Física, F.C.F.M., \\ Universidad de Chile, Santiago 3, Chile \\ 2 Departamento de Física, Facultad de Ciencias \\ Universidad del Bío Bío, Concepción, Chile
}

\section{Introduction}

When studying fluids it is frequently the case that the construction of a reasonable approximate theory becomes an elusive endeavor. The harder and interesting these problems are the better it is to have exact results, coming directly from the microscopic kinetics, both to test the existing theoretical approaches and to gain insights to new ones. To progress in the formulation of fluid dynamics it is desirable to be free from having a precise and realistic description of a particular fluid. The latter is an interesting but different problem.

Hydrodynamics is the main tool to describe fluids from the macroscopic point of view in a wide variety of applications. The basic assumption of hydrodynamics is that changes in a fluid take place smoothly or slowly so that the system can be considered to be in a state of local thermodynamic equilibrium. When the condition of smooth/slow variation is not fully satisfied one can expect the fluid to deviate from the predictions of hydrodynamic calculations. For example, if $X$ is a hydrodynamic field (e.g., temperature) it can be said that $X$ varies smoothly if $\xi=\ell|\nabla X| / X$ is negligible, where $\ell$ is the mean free path of the particles of the fluid or that $X$ varies slowly if $\xi=\tau \frac{\partial X}{\partial t}$ is negligible where $\tau$ is the mean free flight time. The use of kinetic theory becomes essential if $\xi$ cannot be taken to be zero.

Kinetic theory, on the other hand, gives a more fundamental theory but it has been well developed only for rather dilute systems. The first steps in the formulation of kinetic theory reduces the direct hamiltonian dynamics first to Liouville's equation and then to an infinite set of integrodifferential equations, known as the BBGKY hierarchy. Since there is no systematic way 
of applying something like perturbation theory to such system of equations, their analysis has been, and still remains, a patchwork of ingenious techniques that are designed to cover special parameter regimes.

Special mentions deserve Boltzmann's and Enskog's equations. The former can be validated in the so called Boltzmann-Grad limit of low density and finite mean free path, Grad (1958). Enskog's equation has a larger domain of validity and it was originally written for a gas of hard spheres but it can be extended to other cases. For example when the hard spheres interact via a square well potential the collision term on the right hand side of the kinetic equation becomes rather involved but it can be worked out explicitly.

Once one of these kinetic equations is stated the challenge is to find approximate solutions. Perhaps the most widely used method is that of Chapman and Enskog. This method is based on a perturbative expansion about equilibrium where the small parameters are the $r$ th-order spatial derivatives of the density, hydrodynamic velocity and temperature fields. The zeroth order solution is Maxwell's equilibrium distribution, the first order gives Euler's ideal hydrodynamics and the second order provides a version of Navier Stokes equations with linear transport equations and explicit expressions for the transport coefficients. The third order was derived by Burnett in 1935 and will not concern us here.

In hydrodynamics the transport equations are part of the so called constitutive equations in the sense that they are needed to make the hydrodynamic equations a closed (self-contained) system of equations.

Harold Grad in 1958 presented a different way to construct approximate solutions to Boltzmann's (eventually Enskog's) equation, Grad (1958). In his approximate solution he does not expand in gradients of the first five momenta of the velocity distribution (density $n$, hydrodynamic velocity $\mathbf{v}$, and temperature $T$ ) as in the Chapman-Enskog method, but he rather uses a selfconsistent approach additionally involving higher momenta and no gradients of them. In particular he works out in detail the case when the distribution function $f$ is written in terms of $n, \mathbf{v}$ and $T$ and also the traceless and symmetric part of the pressure tensor, $p_{i j}$, and the heat flux vector $\mathbf{q}$, which in $3 \mathrm{D}$ totals 13 momenta ( 8 momenta in 2D and $1+d(d+5) / 2$ in dimension $d$ ). From this method nonlinear transport equations stem naturally.

Decades ago there was no way to separate the tests of new developments in kinetic theory from the challenge to describe particular fluids. Presently it is possible to get semi-experimental results from microscopic computational simulations using molecular dynamic (MD) techniques in which the microscopic interactions are part of the data and they are not bound to being realistic.

These lectures give a partial view of the study of the behavior of simple fluids from a microscopic point of view by means of kinetic theory. See Chapman, Cowling (1970), Hansen, McDonald (1986), Lifshitz, Pitaevskii (1993), Résibois, de Leener (1977), Ferziger, Kaper (1972) combined with the meth- 


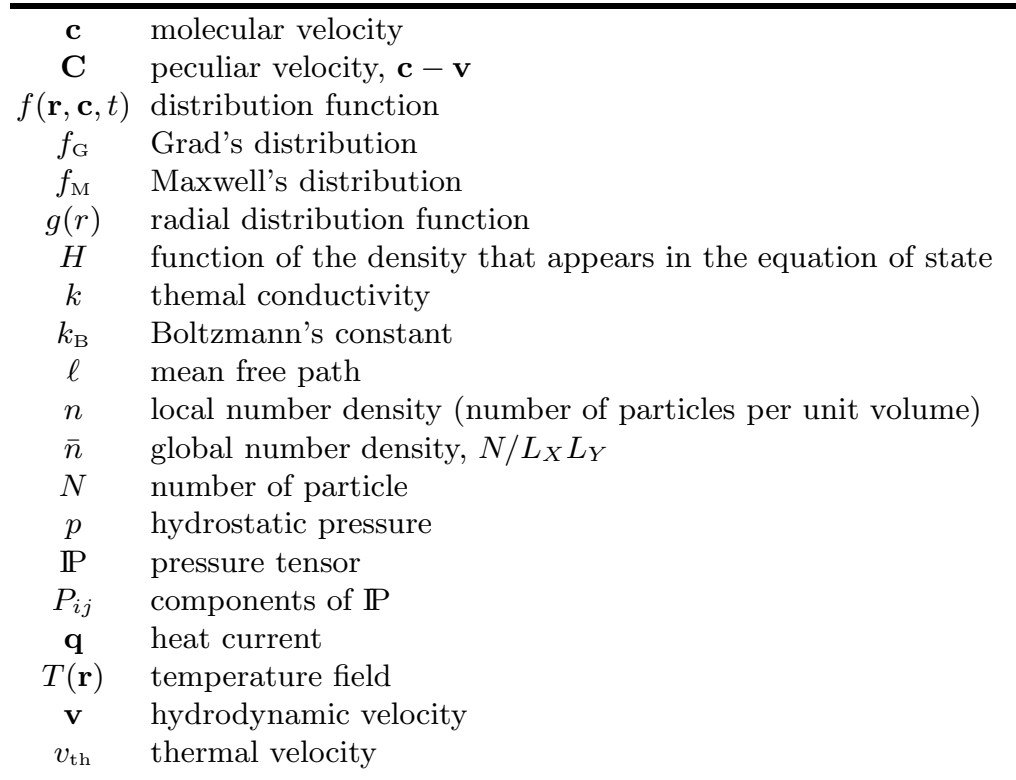

Table 1. Glossary of main latin symbols used in the text.

ods of microscopic computational simulations: Allen, Tildesley (1989), Ciccotti, Hoover (1986), Ciccotti, Frenkel, McDonald (1987) taking advantage of recent efficient algorithms and strategies of molecular dynamics, Rapaport (1980), Lubachevsky (1991), Marín, Risso, Cordero (1993), Cordero, Marín, Risso (1995).

\subsection{Background Generalities on Molecular Dynamics}

Molecular Dynamics (MD), in the sense of these lectures, is a computer simulation technique which traces the microscopic Newtonian time evolution of a systems of $N$ classical particles in the phase space of all of them. In combination with appropriate ensemble or time averaging, the technique gives a solution to the Liouville equation without having to make any assumptions concealed neither behind hydrodynamics nor in the standard formulation of kinetic theory (Bolztmann's, Enskog's or other).

MD simulations offer us a unique opportunity to test every hypothesis that has been made (or is going to be made) about the behavior of fluids. Because of the intrinsic complexities of kinetic theory, MD simulations have become an essential tool. 


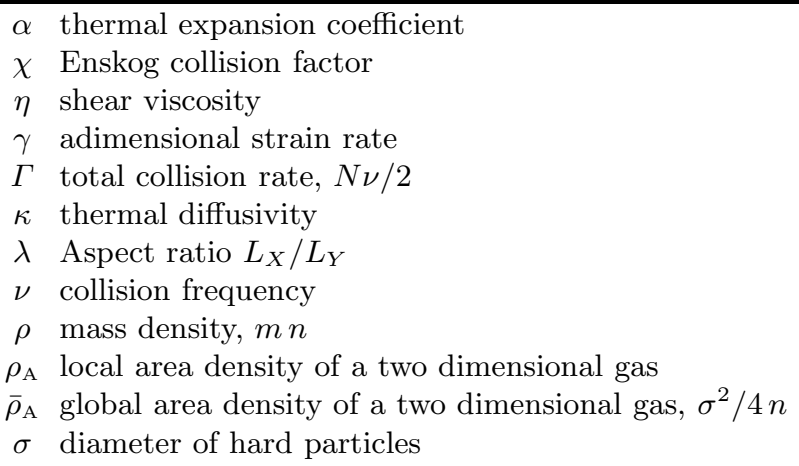

Table 2. Glossary of main greek symbols used in the text.

Making stationary regime MD simulations will usually involve only a few hundred particles and for this reason one has to be careful, because the physics that takes place near the walls may play a non-negligible role. In fact, the fraction of particles that - at any moment - are close to the walls is $\mathcal{O}\left(\frac{1}{\sqrt{N}}\right)$, where $N$ is the total number of particles in the numeric experiment.

It is perhaps interesting to recall that the many important and historical findings were made (by Alder and Wainwright) with systems of about 100 particles mainly at equilibrium. Today many interesting findings involving steady state hydrodynamics behavior are commonly made with systems of about 2000 particles as in Mareschall et al.

The fundamental importance of MD simulations rests in the fact that they provide essentially exact, quasiexperimental data on well defined models. As there is no uncertainty about the form of the interaction potential, theoretical results can be tested unambiguously in a manner that is generally impossible with data obtained in experiments with real fluids. It is also possible to obtain information on quantities of theoretical importance that are not readily measurable in the laboratory.

\subsection{Hard Particle Systems}

Hard Disks. We shall often concentrate in simple bidimensional systems. These systems will consist of $N$ particles of mass $m$ - either bare hard disks or hard disks interacting with a square well potential. The system will be inside a rectangular box of size $L_{X} \times L_{Z}$, aspect ratio

$$
\lambda=L_{X} / L_{Z}
$$




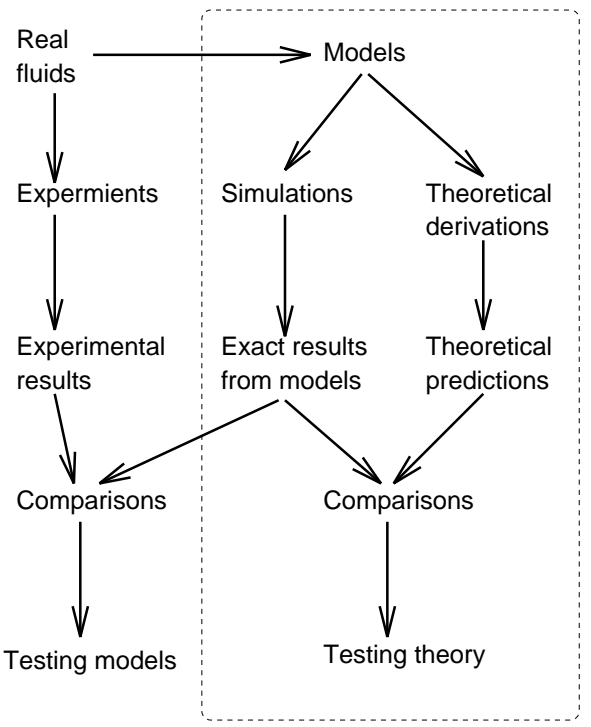

Fig. 1. Diagram adapted from Allen and Tildesley (1989). With realistic interactions it is interesting to compare experiment and simulation results to test a particular model. With any suitable interaction the comparison of the theoretical predictions with the simulational results tests the theory itself. Much work is done within the limits of the dashed line rectangular box above.

The bulk area density is

$$
\bar{\rho}_{\mathrm{A}}=\frac{\pi N \sigma^{2}}{4 L_{X} L_{Z}}
$$

where $\sigma$ is the diameter of the hard core of the particles.

The interaction of the particles with the walls will be discussed in $\S 1.3$.

The equation of state of a system of hard disks has to have the form

$$
p L_{X} L_{Z}=N H\left(\rho_{\mathrm{A}}\right) k_{\mathrm{B}} T
$$

where $\rho_{\mathrm{A}}=\rho_{\mathrm{A}}(\mathbf{r})$ is the local area density. In general the dependence on $T$, on the right hand side, is not trivial, but when the interparticle interaction does not introduce an energy scale a dimensional analysis shows that the pressure has to be proportional to the temperature $T$. The function $H\left(\rho_{\mathrm{A}}\right)$ is as much known as the virial coefficients for this system Kratky (1978) [XX hay otra ref que agreg'o un coef]. A practical and simple approximate expression (Henderson (1975), Barker, Henderson (1976)) is

$$
H\left(\rho_{\mathrm{A}}\right)=\frac{1+\frac{\rho_{\mathrm{A}}^{2}}{8}}{\left(1-\rho_{\mathrm{A}}\right)^{2}}
$$


More accurate equations of state are found in Maeseo, Solana (1993).

The radial distribution function $g(r)$ (defined later in \$1.4) at contact $\chi=g\left(\sigma^{+}\right)$is a function of the density,

$$
\chi\left(\rho_{\mathrm{A}}\right)=\frac{H-1}{2 \rho_{\mathrm{A}}}=\frac{1-\frac{7 \rho_{\mathrm{A}}}{16}}{\left(1-\rho_{\mathrm{A}}\right)^{2}}
$$

and the specific heat coefficients are

$$
c_{v}=\frac{k_{\mathrm{B}}}{m}, \quad c_{p}=\frac{k_{\mathrm{B}}}{m}(1+H(n) \alpha T)
$$

In the last expression $\alpha$ is the thermal expansion coefficient

$$
\alpha=-\frac{1}{n}\left(\frac{\partial T}{\partial n}\right)_{p}^{-1}=\frac{H}{T(n H)^{\prime}}
$$

where the prime indicates derivative with respect to $n$.

A time scale to measure the relaxation time of a system comes from an estimation of the thermal diffusion time accross the system. The temperature diffusion equation is $n k_{\mathrm{B}} \partial T \partial t=-\nabla \cdot \mathbf{q}$. Accepting Fourier's law of heat conduction: $\mathbf{q}=-k \nabla T$, where $k$ is the thermal conductivity, it is seen that $\partial T \partial T=\left(k / n k_{\mathrm{B}}\right) \nabla^{2} T$, which implies a temperature diffusion time for a system in $L \times L$ box:

$$
\begin{aligned}
t_{\text {diff }} & =\frac{n k B L^{2}}{k}=\frac{m c_{p} N}{k} \\
& =\frac{N k_{\mathrm{B}}}{k}
\end{aligned}
$$

\subsection{Characteristics of the Simulations and the Simulator}

The Simulations. The type of simulations we deal with in the present lectures have the following common characteristics.

- the only degrees of freedom of the particles are translational

- the collisions among particles are perfectly elastic, hence conserving momentum and energy

- the diameter of the hard core of the particles is $\sigma$ : the energy potential is infinite for $r<\sigma$

- the particles may additionally interact among themselves with a square potential of depth $-\epsilon$ in the range $\sigma<r<\alpha \sigma, \quad(\alpha>1)$. The parameter $\alpha$ is the adimensional range of the interaction

- the interaction with the walls depends on the case under study. They may be, for example,

o specular collisions (adiabatic slip walls) 
o thermal slip collisions (stress free boundary condition): the particles bounce back conserving their tangential velocity while the normal velocity is sorted out from a Maxwellian distribution characterized by a given temperature $T$.

o thermal nonslip collisions: both components of the velocity are sorted from a heat bath at temperature $T$ and the sign of the tangential component is randomly chosen.

o thermal nonslip moving wall: same as the thermal nonslip wall except that a value $v_{0}$ is added to the tangential velocity to simulate that that wall is moving orthogonal to its normal.

o periodic walls: a particle that hits a wall reenters the system through the opposite wall with the same velocity. In this case there can be collisions between particles which are at different sides of a periodic wall.

o etc

- the basic data to run a simulation are: the number of particles $N$, the aspect ratio $\lambda=L_{X} / L_{Z}$ of the rectangular box and the bulk area density $\bar{\rho}_{\mathrm{A}}$ defined in (2). Other data are needed in each specific class of simulation.

To simulate a fluid one usually wants that the mean free path be significantly smaller that the size of the box, otherwise boundary effects influence the whole system. This condition implies $N \gg 1 / \bar{\rho}_{\mathrm{A}}$. Further, if the simulation is going to be compared with ideal gas results then, loosely speaking, one may say that the mean free path has to be much larger than the size of the particles implying that $\rho_{\mathrm{A}} \ll 1$.

For a gas of disks, the requirement that the equation of state deviates by less than $5 \%$ from the ideal gas equation $(H=1)$ implies that the area density satisfy $\bar{\rho}_{\mathrm{A}} \leq 0.025$. Additionally requiring that, for a system in a $L \times L$ square box , the the mean free path satisfies $\ell / L \leq 0.05$ yields that the number of particles has to be $N \geq 1570$.

The Simulator. In the following we describe a strategy to make efficient simulations of systems of several thousand particles interacting via a piecewise constant potential. A performance of several million collisions per hour is perfectly attainable in present day standard workstations. These algorithms are particularly appropriate for kinetic studies at a microscopic level in situations near or far from thermodynamic equilibrium.

In the case of piecewise constant potentials the particles move free of each other except at discrete times when they suffer impulsive forces or events. The evolution of each particle between events follows Newton's equations of motion with whatever external field (e.g., gravity) may exist but free from interparticle interactions. The events take place whenever a particle hits one of the steps of the potential energy function. Applications of this type of 
simulations are illustrative and inspiring since the beginning of molecular dynamics till present days. The basic steps of a simulation of this type are sketched in Fig. 2.

0a) An initial state is given, namely positions and velocities for all particles and

0b) the list of all possible future events, or FEL (future event list), is initialized.

1) The next event is determined from the FEL;

2 ) if necessary, positions and velocities are updated to the instant of this event;

3) the collision rule that defines the model is applied to the state of the particle(s) involved in the event;

4) new events have to be predicted for the particles that have been involved in the present event;

5) the new predicted events are inserted (scheduled) into the FEL and go back to step (1).

Fig. 2. Basic cycle in an event driven simulation.

A typical action at step 2 for particles moving in the presence of an acceleration of gravity $\mathrm{g}$ could be

$$
\begin{aligned}
& \mathbf{r}_{k} \longleftarrow \mathbf{r}_{k}+\mathbf{v}_{k} \tau_{k}+\frac{1}{2} \mathbf{g} \tau_{k}^{2} \\
& \mathbf{v}_{k} \longleftarrow \mathbf{v}_{k}+\mathbf{g} \tau_{k}
\end{aligned}
$$

where $\tau_{k}$ is the time interval between the previous event and the present one.

This type of dynamics is called event driven, because it proceeds by time steps $\tau_{k}$ that are dictated by the dynamics itself instead of proceeding via time steps $\delta t$ predefined in the algorithm as it is done whenever continuously varying potentials are used. The enormous advantage of event driven simulations is that - because of their efficiency - it is possible to explore significant parts of the evolution of the system.

One of the important bottlenecks of these simulations could be step (1) unless the next event is determined through a carefully written algorithm. A breakthrough in efficiency was given by Rapaport (Rapaport (1980)). A different strategy which is efficient in a wider range of densities is found in Marín, Risso, Cordero (1993).

The FEL is important because - as we have argued - these simulations proceed jumping analytically from one collision (or event) to the next. And 
the next event is determined using a binary tree as part of the FELs structure. But since predictions have to be updated as the simulation proceeds the binary tree is not only called for to pickup the next event but also when new information has to be inserted in it. This may be costly. In our strategy we have been able to reduce the number of accesses to the binary tree to about one access for every particle involved in each collision (Marín, Risso, Cordero (1993)). We know of no other algorithm which can attain this performance. Some improvements were introduced in Marín, Cordero (1995).

Cells and a new type of event. To determine the next event without risking the possibility of a mistake it is apparently necessary to know first the times when every possible collision would take place. This means particle-particle as well as particle-wall events. There are $\mathcal{O}\left(N^{2}\right)$ such times and they would have to be compared to get the smallest one. In typical situations most of the predicted events never happen since the particles involved change their direction of movement at previous collisions. In this sense we could say that many predicted events become invalidated.

Since the beginning of the history of event driven simulations the convenience of dividing the system in cells was clear (Alder, Wainright (1959)). A particle belongs to a cell if its center is inside the cell. The linear size $D$ of the cells is chosen so that a particle cannot interact but with particles which are in its own cell or in the neighboring cells. For example, in a bidimensional system broken in square cells a typical cell is surrounded by eight others. The neighborhood of a particle $k$ is the cell where $k$ is plus the set of neighboring cells.

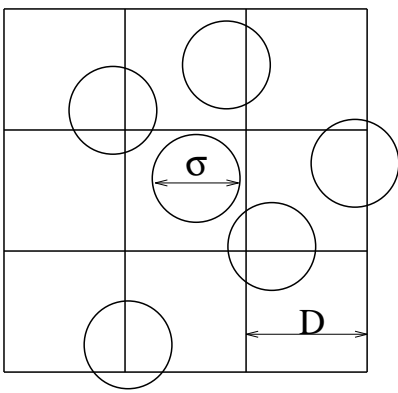

A particle $a$ belongs to the cell where its center is. The neighborhood of $a$ will usually contain 9 cells.

For hard disks of diameter $\sigma$, for example, the linear size $D$ of a cell has to satisfy, $D>\sigma$ to make the previous concepts consistent.

A new type of event is introduced: crossing a cell wall. This event has no physical meaning of course. Their occurrence does not alter the physical evolution of the system but it can be realized that now there is no need to predict events involving a particle $i$ and objects (other particles or a wall) beyond its neighborhood since there is at least one event involving $i$ and an object in its neighborhood prior to any event with objects outside it.

To make this point clear suppose the rare situation when the next physical event involves the collision between two particles that are far beyond their respective neighborhoods. From the algorithms point of view this event is preceded by several cell wall crossings. Each one of these events - artifacts 
of the algorithm - implies that at step 4 above, new events are predicted for one of these particles. After a sequence of cell wall crossings the two particles will see each other and the actual collision will correctly be predicted.

Hence predictions for each particle $i$ at step 4 are made only between objects belonging to the neighborhood of $i$. After $i$ suffers a collision all predictions for it have to be renewed whereas if $i$ makes a cell wall crossing its neighborhood changes and new predictions are added: those involving $i$ and objects in the new cells belonging to the neighborhood of $i$.

Since each neighborhood contains a number of particles which is independent of the size of the system, the total number of events that have to be predicted for the whole system is $\mathcal{O}(N)$.

The five steps mentioned above have to suffer some minor obvious modifications because this new type of event is introduced. For example, if the present event is a cell wall crossing there is no need to apply any collision rule.

The future event list (FEL). To search for the next event efficiently one has to keep future events in order. Binary search is a standard strategy, Knuth (1973). For example when looking for a word in a dictionary we proceed roughly as follows: we cut the dictionary in two equal halves, make a comparison to decide in which half is the target word, then break the chosen part in two again and so forth. The search time is $\mathcal{O}\left(\ln _{2} N\right)$ if there are $N$ words in the dictionary.

There are many variants of the strategy sketched above. Which strategy should be chosen depends on other aspects of the problem. In the case of our simulations three functions act on the FEL: (a) getting the next event; (b) inserting new predicted events and (c) erasing invalidated events. Binary trees in general allow for efficiently implementing the operations (a) and (b), but erasing is not trivial and it may be time consuming. A careful assessment of these problems and experimenting with some of the structures put forward in the literature we chose, in Marín, Risso, Cordero (1993), the structure that we pass to describe. It will be seen that with our algorithm there is no need to make eliminations within the binary tree.

For each particle $i$ is associated a single linked list $L_{i}$ containing the future events $\mathcal{E}_{i}(x)$ predicted for particle $i$ with an object $x$ (another particle or a wall). When an event $\mathcal{E}_{i}(k)$ is predicted for particle $i$ at step 4 , it is inserted in the list $L_{i}$ and it is not inserted in the list $L_{k}$. Namely, each future event is entered only once to the FEL. These $\mathcal{E}_{i}(x)$ are structured variables containing the necessary information associated to the specific event. In particular they contain the time when the event is scheduled to happen.

Each one of these $N$ lists has a local minimum event, the event in the list that has the lesser schedule time. These $N$ local minima are the only events that enter the binary tree. The determination of the local minimum for particle $i$ and its insertion into the binary tree is performed regardless of the existence of other possible events $\mathcal{E}_{\ell}(i)$ within the FEL. 
The binary tree structure that we use is that of a complete binary tree (Knuth (1973)) or CBT. It is a binary tree of $K$ levels. At level 0 is the root, at level 1 are its two children, at level $n$ there are $2^{n}$ nodes, children of the nodes at the previous level. $K$ is determined by the number $N$ of particles: $2^{K-1}<N \leq 2^{K}$, so that the lowest nodes (leaves) are exactly $N$ and they are partly at the incomplete level $K$ and the rest at level $K-1$. Each particle is associated to one and only one of these lowest nodes or leaves once and for ever.
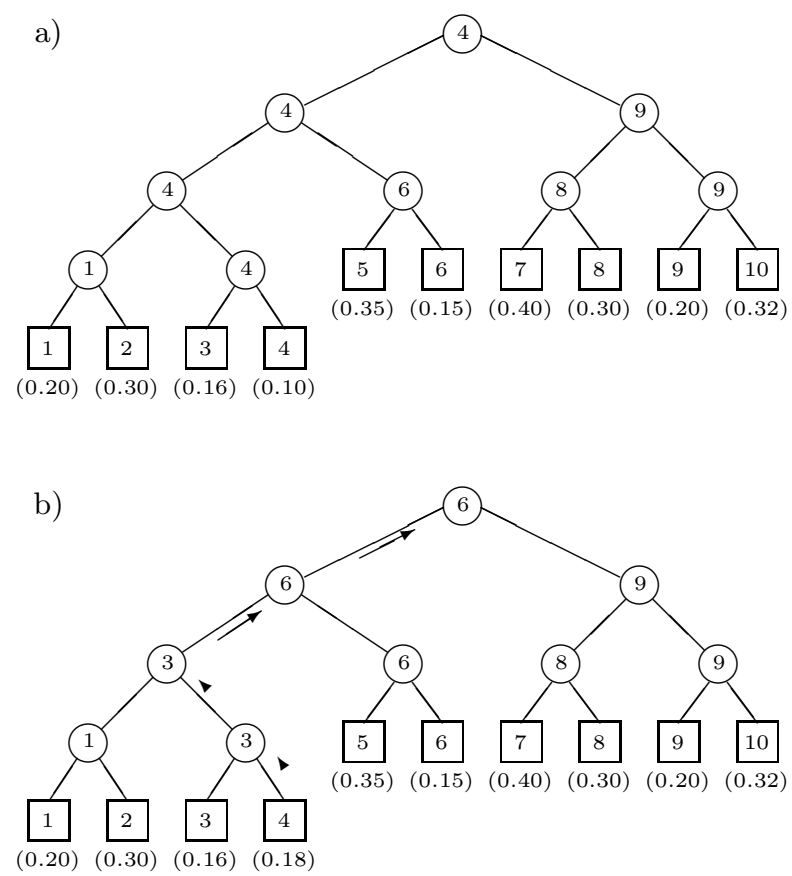

Fig. 3. a) Complete binary tree (CBT) for 10 particles. The local minima times are in parenthesis. b) The CBT upgraded after the local minimum of particle 4 was changed to the value 0.18 .

Since only the local minima enter the CBT, each particle-label in it has associated the time at which the corresponding local minimum is scheduled to happen. The logic of the CBT is the same one used in many sports tournaments: to each competitor there is associated a fixed leaf node. The name of the winner of each individual match (i.e. comparison of the associated 
times) is transcribed to the parent node. The same rule is recursively applied upwards in the tree and in this way each internal node has the label of the particle with lesser time of its two children. The label of the absolute winner reaches the root of the tree. This is the particle whose local minimum should be the next event. During the simulation the form of the CBT and the labels of the leaves remain unaltered, while the labels at the internal nodes change dynamically.

When a particle $i$ suffers a collision, its list $L_{i}$ is erased and replaced by the list of new predictions involving $i$. This is done at step 4. On the contrary, if particle $i$ crosses a cell wall, then its neighborhood changes. Events involving $i$ with other objects in the new cells are added to the list $L_{i}$.

In the two situations described in the previous paragraphs it is necessary to reobtain the local minimum for $i$ which is then inserted into the binary tree replacing the old one. Matches are performed along the natural path from leaf $i$ towards the root of the CBT. It is easy to check that $\mathcal{O}(\ln N)$ matches are performed as part of step 5 .

At this point it is necessary to clarify that only the labels $i$ identifying the particles associated to the local minima enter the binary tree and not the full structures $\mathcal{E}_{i}(x)$. Inserting and deleting is efficient because it is an operation over the lists $L_{i}$. In fact we have established in Marín, Risso, Cordero (1993) that the cost of picking the next event and scheduling $n$ new events for each particle are $\mathcal{O}(1)$ and $\mathcal{O}(n-1+\ln N)$ respectively. That is, we have been able to reduce the number of accesses to the binary tree to about one access for every particle involved in each collision. As we said before, we know of no other algorithm which can attain this performance.

In the case of continuos potentials a totally different strategy has to be followed. Typically they are based on Verlet's algorithm. A quite recent reference from which the literature can be traced back is Glikman et al (1996)

Measurements. The system has to be relaxed before starting measurements in a study of stationary regimes.

To make observations during the simulations we have divided the system in cells to observe its behavior. These cells must not be confused with the cells of the central algorithm. The routines make a careful balance of mass, momentum and energy in each cell (these are densities) and what comes in or out across each wall of every cell (integrated fluxes). The measured quantities are then averaged in time in every cell or wall depending on whether they are densities or fluxes.

Nomenclature: We distinguish a flux vector $\mathbf{J}$ (which could also be called a current density) from an integrated flux across a surface $\mathcal{S}, \Phi=\int \mathbf{J} \cdot d \mathcal{S}$. But when no confusion is possible the word flux is used to mean either a flux vector or an integrated flux. 
Measuring densities. In principle this means evaluating

$$
\bar{\varphi}=\frac{1}{\tau} \int_{t_{0}}^{t_{0}+\tau} \varphi d t
$$

during the simulation. Since our simulations are driven by discrete events these averages are in practice evaluated in the form

$$
\bar{\varphi}=\frac{1}{\tau} \sum_{k} \int_{t_{k-1}}^{t_{k}} \varphi d t
$$

where the $t_{k}$ are a subset of the event times that we shall call the interesting events associated to $\varphi$. To understand the idea of an interesting event we give two examples.

To determine the density in a cell $A$ we want to average the number of particles in $A$. With this aim the only interesting events are those corresponding to particles crossing from one cell to the next. No collision event is interesting in this case. The integrals appearing in (10) trivially reduce to $m_{k-1}\left(t_{k}-t_{k-1}\right)$.

To determine the average kinetic energy in every cell, in the case of a system of bare hard disks, all events are interesting except for the collisions that occur between particles belonging to the same cell.

Measuring fluxes. To get an integrated flux, $\Phi_{w}=\int_{w} \mathbf{J} \cdot d \mathcal{S}$, across a cell walls $w$ it is necessary to keep track of every exchange, per unit time, of mass, momentum and energy across the wall $w$. The instantaneous flux of a quantity $\varphi$ at the time $t_{k}$ is given by $\varphi\left(t_{k}\right) \delta\left(t-t_{k}\right)$, where $\varphi$ is the magnitude of the transfered property. Averaging in time yields

$$
\Phi_{w}(\varphi)=\frac{1}{\tau} \int_{t-\tau}^{\tau} \varphi\left(t_{k}\right) \delta\left(t-t_{k}\right) d t=\frac{1}{\tau} \sum_{k} \varphi\left(t_{k}\right)
$$

These exchanges can occur simply because a particle crosses from one cell to the next contributing to the kinematic transport, or they occur because there is a collision involving particles which are in different cells (exchanging momentum, for example) contributing to collisional transport. The simulation can keep separate track of the kinetic and collisional contributions of every flux vector being studied. 
Special care has to be taken when the exchange $\varphi\left(t_{k}\right)$ occurs between two particles that belong to cells which only share a corner as $A$ and $D$ in the figure. This is a problem because fluxes. need to be defined through walls and not corners. In the figure we represent the collision of two particles belonging to diagonally neighboring cells $A$ and D.

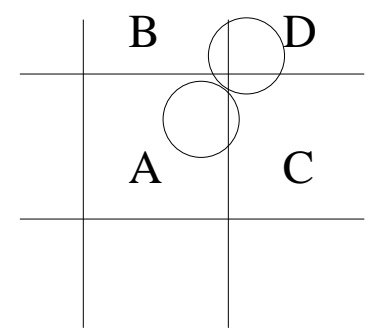

Collision between two particles that belong to cells that share only a corner.

\subsection{The Radial Distribution Function}

For completeness sake we have included this section where tha radial distgribution function and Enskog's collision factor $\chi$ are defined.

In equilibrium statistical mechanichs it is standard to define distribution functions $\rho_{N}^{(\nu)}\left(\mathbf{r}_{1}, . . \mathbf{r}_{\nu}\right)$ normalized to

$$
\int \rho_{N}^{(\nu)}\left(\mathbf{r}_{1}, . . \mathbf{r}_{\nu}\right) d \mathbf{r}_{1} . . d \mathbf{r}_{\nu}=\frac{N !}{(N-\nu) !}
$$

of which $\frac{1}{N} \rho_{N}^{(1)}(\mathbf{r})$ is the probability density to find a particle about the position $\mathbf{r}$. Similarly $\frac{2}{N(N-1)} \rho_{N}^{(2)}\left(\mathbf{r}, \mathbf{r}^{\prime}\right)$ is the probability density of having one particle about $\mathbf{r}$ and another about $\mathbf{r}^{\prime}$. From these first two functions the pair correlation function is defined as

$$
g_{N}^{(2)}\left(\mathbf{r}_{1}, \mathbf{r}_{2}\right)=\frac{\rho_{N}^{(2)}\left(\mathbf{r}_{1}, \mathbf{r}_{2}\right)}{\rho_{N}^{(1)}\left(\mathbf{r}_{1}\right) \rho_{N}^{(1)}\left(\mathbf{r}_{2}\right)}
$$

For an isotropic fluid this last function depends only on the magnitud of the relative position vector $r=\left|\mathbf{r}_{1}-\mathbf{r}_{2}\right|$. In such case the function $g(r)$ is known as the radial distribution function. It can be seen that for large $r$ the function $g$ tends to $1+\frac{1}{N}$. The radial distribution function has a typical oscilating form and it can be found in many textbooks on statistical mechanics.

For hard spheres of diamter $\sigma$ the value of $g$ at contact, namely $g\left(\sigma^{+}\right)$is called the Enskog collision factor $\chi$,

$$
\chi=g\left(\sigma^{+}\right)
$$

and it depends on the density of the system and therefore on position. If the potential is less trivial $\chi$ depends on the temperature as well. This factor $\chi$ will appeare in many expressions starting from $\S 2$. 
In in kinetic theory one encounters integrals of the form

$$
I=\int_{0}^{\infty} \phi^{\prime}(r) g(r) F(r) m d r
$$

for which it is often useful to define the auxiliary function

$$
y(r)=e^{-\beta \phi} g(r)
$$

where $\beta=1 / k_{\mathrm{B}} T$ and $\phi$ is the interparticle potencial, for then the above integral can be transformed to

$$
\begin{aligned}
I & =\int_{0}^{\infty} \phi^{\prime} e^{-\beta \phi} g(r) F(r) d r \\
& =-\frac{1}{\beta} \int_{0}^{\infty} g(r) F(r) \frac{d}{d r} e^{-\beta \phi} d r
\end{aligned}
$$

but if the potential $\phi(r)$ is a hard sphere potential (zero or infinity), then the exponential in the integrand is a step function and its derivative is a delta-function $\delta(r-\sigma)$. Hence $I$ can be writtten as

$$
I=-k_{\mathrm{B}} T \chi F(\sigma)
$$

\subsection{Problems}

The best way to write good programs is to write straightforward routines without any attempt to be clever. Never mind if it is slow and uses lots of memory. Once you have checked that it works as it is supposed to work do not touch it again. If you want to improve it work over a copy with a different name.

1. Write a program for the $1 \mathrm{D}$ movement of two dot particles of masses $m_{1}$ and $m_{2}$ respectivelly, moving in a straight line under the effect of gravity $g$. They have perfectly elastic collisions among themself and particle 1 hits the floor elastically as well. Normalize you variables so that the total energy of the system is $E=1$ and $R=m_{2} / m_{1}$. Plot $x_{2}$ against $v_{2}$ every time particle 1 hits the floor. Notice that these points are always within a parabola. Use, for example, $R=3$. Different initial conditions may lead to different graphs. Carefully explore the graphs for a wide class of initial conditions.

2. Extend the previous program for $N$ hard rods of length $a$, unit mass, moving inside the box: $[x=0, x=L]$ and without gravity. Take $N=20$, $N a / L=0.75$ and make a time average of the local density within every interval $L / 100$ long. Also determine the average local density relative to the position of a particle up to $4 a$ away from it. These densities have a structure. The reason for this is that the probability of having a particle in a given position depends on the positions of the others. This is the basic idea of correlations in position. To write the measurement routines take into consideration what was said under Measuremets in $\S 1.2$. 
3. Consider just one particle moving in a straight line under de effect of gravity, $g$. The floor moves following $z(t)=A \cos (\omega t)$ and the particle bounces back from the floor with velocity $v^{\prime}=(1+\eta) \dot{z}-\eta v$ where $0<\eta<1$ is the restitution coeefficient and $v$ is the velocity with which the particle hits the floor. Besides $\eta$ the other important parameter is $G=\frac{A \omega^{2}}{g}$. For every value of $G$ take many different initial conditions and see whether asymptotically the particle looses all its energy and sticks to the floor or continues bouncing for ever. For a fixed value of $\eta$ (e.g., $\eta=0.6$ ) plot against $G$ all the possible values of $v^{\prime}$ when the asymptotic regime is a bouncing particle. You will discover a complex structure, interprete it.

4. Write a program for the evolution of a 2D system of hard disks of diameter $\sigma$. To be able write the future event list of times when any two disks $a$ and $b$ will hit each other define from the positions and velocities at time $t_{0}$ the quantities $\mathbf{r}=\mathbf{r}_{a}-\mathbf{r}_{b}, \mathbf{v}=\mathbf{v}_{a}-\mathbf{v}_{b}$ and $b=\mathbf{r} \cdot \mathbf{v}$. The two disks will hit each other at time $t_{1}$ if $\Delta=b^{2}-v^{2}\left(r^{2}-\sigma^{2}\right)$ is positive,

$$
t_{1}=t_{0}-\frac{b+\sqrt{\Delta}}{v^{2}}
$$

and $t_{1}>t_{0}$. Otherwise set $t_{1}=\infty$. For the rest use the scheme describen in Fig.2.

\section{Primer on Kinetic Theory}

\subsection{The One Particle Distribution Function}

Consider a fluid composed of $N$ unstructured particles. The one particle state space $\Gamma_{1}$ will be described with either position and momentum $(\mathbf{r}, \mathbf{p})$ or position and velocity $(\mathbf{r}, \mathbf{c})$. This is a six dimensional space. Divide the space in small cells labeled by $\xi$ with volume $\Delta$. Considering many macroscopically equal replicas of the system, call $\mathcal{N}(\xi, t)$ the expected number of particles to be found in cell $\xi$ at time $t$. For any reasonable fluid these numbers will decrease exponentially as the velocity coordinates increase. If the cells $\Delta$ are sufficiently small these occupation numbers, normalized to $\Delta$ through,

$$
f(\xi, t)=\frac{\mathcal{N}(\xi, t)}{\Delta}
$$

partly describe the system in great detail. In the limit of very small cells we get a distribution function $f(\mathbf{r}, \mathbf{c}, t)$ normalized to the number density $n(\mathbf{r}, t)$,

$$
\begin{gathered}
\int_{\Gamma_{1}} f(\mathbf{r}, \mathbf{c}, t) d^{3} c=n(\mathbf{r}, t) \\
\int n(\mathbf{r}, t) d^{3} r=N
\end{gathered}
$$


Even though $f$ gives much information about the system it is far from giving a complete description of it. It does not describe, for example, the correlations that usually exist in a fluid as those describen in $\S 1.4$. Formally

$$
f(\mathbf{r}, \mathbf{c}, t) d^{3} r d^{3} c
$$

gives the number of particles expected about the point $(\mathbf{r}, \mathbf{c})$ of $\Gamma_{1}$ at a given instant $t$. The distribution $f$ is useful to evaluate averages of one particle quantities. The average is a macroscopic quantity $\langle\varphi\rangle(\mathbf{r}, t)$ :

$$
\langle\varphi\rangle(\mathbf{r}, t)=\frac{1}{n(\mathbf{r}, t)} \int \varphi(\mathbf{r}, \mathbf{c}, t) f(\mathbf{r}, \mathbf{c}, t) d^{3} c
$$

In this way one defines the hydrodynamic velocity $\mathbf{v}(\mathbf{r}, t)$ and the peculiar velocity $\mathbf{C}(\mathbf{r}, t)$

$$
\mathbf{v}(\mathbf{r}, t)=\frac{1}{n(\mathbf{r}, t)} \int \mathbf{c} f(\mathbf{r}, \mathbf{c}, t) d^{3} c, \quad \mathbf{C}(\mathbf{r}, t)=\mathbf{c}-\mathbf{v}(\mathbf{r}, t)
$$

\begin{tabular}{|c|c|c|}
\hline$X$ & $\langle X\rangle$ & name \\
\hline $\mathbf{c}$ & $\mathbf{v}(\mathbf{r}, t)$ & hydrodynamic velocity \\
\hline$\frac{1}{2} C^{2}$ & $u_{\mathrm{K}}(\mathbf{r}, t)$ & kinetic energy per unit mass \\
\hline$\rho C_{i} C_{j}$ & $\left(\mathbb{P}_{\mathrm{K}}\right)_{i j}$ & kinetic part of the pressure tensor \\
\hline$\frac{1}{2} \rho C^{2} \mathbf{C}$ & $\mathbf{q}_{\mathrm{K}}$ & kinetic part of the heat flux vector \\
\hline
\end{tabular}

Table 3. First momenta of the distribution function

For a gas at equilibrium in $d$ dimensions the distribution function is

$$
f_{\mathrm{M}}(\mathbf{r}, \mathbf{c}, t)=n(\mathbf{r}, t)\left(\frac{m}{2 \pi k_{\mathrm{B}} T(\mathbf{r}, t)}\right)^{d / 2} e^{-m C(\mathbf{r}, t)^{2} / 2 k_{\mathrm{B}} T(\mathbf{r}, t)}
$$

but in general the distribution function $f$ deviates from $f_{\mathrm{M}}$ and we will see examples where this deviation has interesting measurable implications. 
From the notion of $f$ it follows, for example, the hystogram of the number of particles with rapidity about $C=\equiv|\mathbf{C}|$ is proportional to $C^{d-1} f_{\mathrm{M}}$. A $2 \mathrm{D}$ case at equilibrium is seen in Fig. 4.

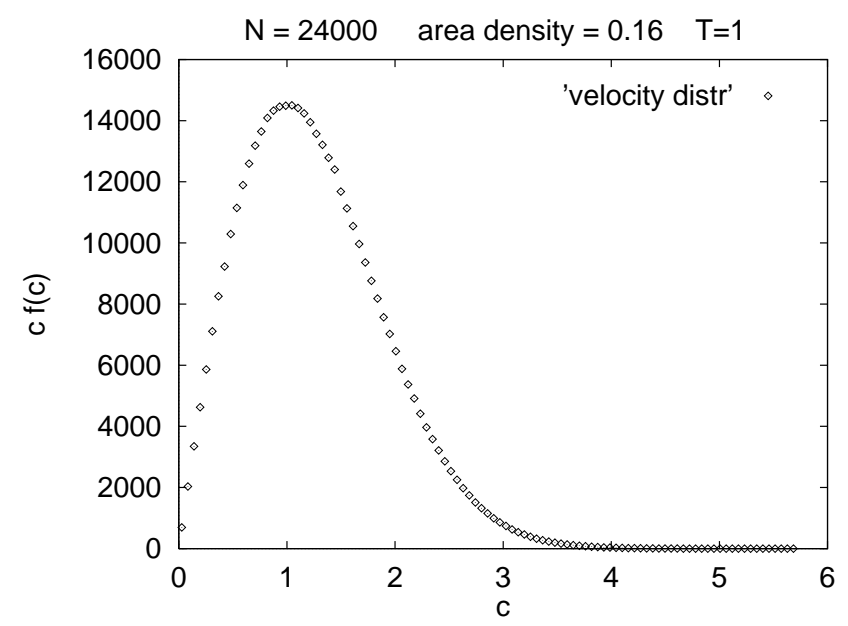

Fig. 4. Hystogram of the number of particles having rapidity between $C$ and $C+d C$ in a $2 \mathrm{D}$ system at equilibrium. In the present case the figure corresponds to a system of 24000 hard disks and area density 0.16 simulated in a personal computer. Starting with a relaxed system, the figure correspondes to an average over 60 million collisions.

Formally we will often identify the temperature field $T(\mathbf{r}, t)$ as it is understood from quasiequilibrium statistical mechanics: it is related to $u_{\mathrm{K}}$ through

$$
u_{\mathrm{K}}(\mathbf{r}, t) \equiv\left\langle\frac{1}{2} C^{2}\right\rangle=\frac{d}{2 m} k_{\mathrm{B}} T(\mathbf{r}, t)
$$

In general the momenta of $f(\mathbf{r}, \mathbf{c}, t)$ define interesting physical quantities, as in table 3. Since these momenta are local averages - in the sense that $\mathbf{r}$ is kept fixed - they can be thought of as densities.

\subsection{Mean Free Path}

A textbook illustration of the use of the notion behind (22) let us see how to make a rough estimate of the collision frequency in a gas of spheres of diameter $\sigma$. Consider a point $\left(\mathbf{r}, \mathbf{c}_{1}\right)$ of $\Gamma_{1}$. There are $f\left(\mathbf{r}, \mathbf{c}_{1}, t\right) d^{3} r d^{3} c_{1}$ particles about it and let us think of them as the target. Particles that will collide with this target in the interval $(t, t+d t)$ and having velocity about $\mathbf{c}_{2}$ have to be at $t$ inside a cylinder with section $\pi \sigma^{2}$ and length $\left|\mathbf{c}_{2}-\mathbf{c}_{1}\right| d t$. The number 
of particles about $\mathbf{c}_{2}$ inside this cylinder is $\pi \sigma^{2}\left|\mathbf{c}_{2}-\mathbf{c}_{1}\right| d t f\left(\mathbf{r}, \mathbf{c}_{2}, t\right) d^{3} c_{2}$. We are evaluating both distributions at the same point because we are assuming that the mean free path is much larger than $\sigma$. For simplicity sake's sometimes it is assumed that the two probabilities are independent, but they are not. As a first approximation Enskog realized that it is necessary to add a corrective factor which, in a reasonable approximation, is the Enskog factor $\chi(\mathbf{r})$, namely, the radial distribution function at contact described in $\S 1.4$. Hence, the expected number of collisions per unit time and volume is

$$
\pi \sigma^{2} \chi(\mathbf{r}) \int f\left(\mathbf{r}, \mathbf{c}_{1}, t\right) f\left(\mathbf{r}, \mathbf{c}_{2}, t\right)\left|\mathbf{c}_{2}-\mathbf{c}_{1}\right| d^{3} c_{1} d^{3} c_{2}
$$

and the collision frequency $\nu$ per particle is then

$$
\nu=\frac{\pi \sigma^{2}}{n(\mathbf{r}, t)} \chi(\mathbf{r}) \int f\left(\mathbf{r}, \mathbf{c}_{1}, t\right) f\left(\mathbf{r}, \mathbf{c}_{2}, t\right)\left|\mathbf{c}_{2}-\mathbf{c}_{1}\right| d^{3} c_{1} d^{3} c_{2}
$$

The mean time between collisions is $\tau=\nu^{-1}$ and the mean free path is

$$
\ell=\tau v_{\text {th }}
$$

where $v_{\mathrm{th}}=\langle|\mathbf{c}|\rangle$ is the thermal velocity.

\begin{tabular}{|c|c|c|}
\hline dimension & $v_{\mathrm{th}}$ & $\ell$ \\
\hline 2 & $\sqrt{\frac{\pi k_{\mathrm{B}} T}{2 m}}$ & $\frac{1}{2 \sqrt{2} \chi n \sigma}=\frac{\pi \sigma}{8 \sqrt{2} \chi \rho_{\mathrm{A}}}$ \\
\hline 3 & $2 \sqrt{\frac{2 k_{\mathrm{B}} T}{\pi m}}$ & $\frac{1}{\sqrt{2} \chi n \pi \sigma^{2}}=\frac{\pi \sigma}{6 \sqrt{2} \chi \rho_{\mathrm{V}}}$ \\
\hline$d$ & $\sqrt{\frac{2 k_{\mathrm{B}} T}{m} \frac{\Gamma\left(\frac{d+1}{2}\right)}{\Gamma\left(\frac{d}{2}\right)}}$ & $\frac{\Gamma\left(\frac{d+1}{2}\right)}{\sqrt{2} \chi n \sigma^{d-1} \pi^{(d-1) / 2}}$ \\
\hline
\end{tabular}

Table 4. Approximate expressions for the thermal velocity and mean free path for a gas of hard spheres in diferent dimensions when the distribution is Maxwellian.

The total collision rate for a system of $N$ particles is

$$
\Gamma=\frac{N}{2} \nu=\frac{N}{2} \frac{v_{\text {th }}}{\ell}
$$


and, in case of local thermodynamic equilibrium can be calculated with the expressions in Tab.4

The total number of collisons in one thermal diffusion time is $N_{\text {diff }}=$ Gammat $_{\text {diff }}$ which, for a dilute gas turns out to be

$$
N_{\text {diff }}=2 N^{2} \chi \rho_{\mathrm{A}}
$$

Since the computational time for every collison in $\mathcal{O}(\ln N)$ running a system of hard disks for one diffusion time takes a CPU time $\mathcal{O}\left(N^{2} \ln N\right)$ putting a stringent cost for simulating large systems.

DAR VALORES DEL MUNDO REAL PARA $\ell, \nu$ ETC. XX

\subsection{On Fluxes and Flux Vectors}

Balance equations connect flux vectors $\mathbf{J}$ with densities. The flux vector associated to a microscopic quantity $\varphi(\mathbf{r}, \mathbf{c}, t)$ can be defined with respect to an absolute frame of reference or with respect to the frame that moves with the hydrodynamic velocity $\mathbf{v}(\mathbf{r}, t)$. The latter is a more interesting quantity but both are trivially related. The kinetic contribution to the flux vector associated to a quantity $\varphi$ in the co-moving frame is

$$
\mathbf{J}_{\varphi}^{(K)}(\mathbf{r}, t)=\int \varphi(\mathbf{r}, \mathbf{c}, t) f(\mathbf{r}, \mathbf{c}, t) \mathbf{C}(\mathbf{r}, t) d^{3} c
$$

In a one componet fluid there is no co-moving mass flux. The kinetic contribution to the momentum flux vector is obtained choosing $\varphi=m C_{i}$ and defining a tensorial quantity that was already mentioned in table 3 ,

$$
\mathbb{P}_{\mathrm{K}}(\mathbf{r}, t)=m \int \mathbf{C ~ C ~} f(\mathbf{r}, \mathbf{c}, t) d^{3} c
$$

For gases usually all flux vectors which are not of kinetic origin can be neglected, and therefore assume, for example, that $\mathbb{P}=\mathbb{P}_{K}$. The hydrostatic pressure is

$$
p=\frac{1}{3} \operatorname{Tr} \mathbb{P}
$$

hence the ideal gas equation of state is satisfied,

$$
p=n k_{\mathrm{B}} T
$$

The flux vector of the kinetic energy ( choosing $\varphi=\frac{m}{2} C^{2}$ ) also defines a quantity that we already know

$$
\mathbf{q}_{\mathrm{K}}=\frac{m}{2} \int C^{2} \mathbf{C} f(\mathbf{r}, \mathbf{c}, t), d^{3} c
$$

The total macroscopic fluxes have further contributions. Consider, for example, an imaginary surface element $d^{2} \mathcal{S}$ cutting the fluid. Whenever a particle crosses that surface element there is a kinetic contribution to some 
fluxes. Parenthetically, we say that a particle crosses a surface when its center does so.

If two particles - coming from opposite sides of the surface element $d^{2} \mathcal{S}$ - hit each other and then continue their flight without crossing $d^{2} \mathcal{S}$ there is a contribution to the fluxes, but it is no longer a kinetic contribution but rather a collisional one. It may also happen that two bound particles cross $d^{2} \mathcal{S}$ In that case there is a contribution to the potential energy flux. It is seen then, that fluxes have contributions which can be either of kinetic origin or due to the interactions. In the case of the energy flux (the heat flux) the interaction implies two contributions: one due to collisions and one that occurs when there are bound particles. In gases at normal pressure the kinetic transport dominates with no competition. Hence they behave as "ideal gases". In dense gases and liquids the different contributions compete and in solids the kinetic transport is negligible.

The concept of a heat flux vector is perhaps intuitive but the physical idea behind the components of $\mathbb{P}$ is less so. A careful analysis shows that $P_{i i}$ is the force per unit area in the direction $i$ exerted over the plane perpendicular to the direction $i$. For this reason the $P_{i i}$ are called normal pressures. A non diagonal term $P_{i j}$ is a shear tension in the sense that it represents the force per unit area in the direction $i$ exerted over the plane perpendicular to the direction $j$. The vector $\left(P_{i 1}, P_{i 2}, P_{i 3}\right)$ is the net force per unit area perpendicular to direction $i$.

Mean Free Path Estimates Let us illustrate the evaluation of a momentum flux vector using intuitive but rough argumets based on the notion of mean free path. Consider a gas between to parallel plates perpendicular to the direction $\hat{z}$. The upper plate moves with velocity $v_{0} \hat{x}$ and the lower one moves with velocity $-v_{0} \hat{x}$. If $v_{0}$ is small enough the system stabilizes in a regime characterized by a hydrodynamic velocity field $\mathbf{v}=\left(v_{x}(z), 0,0\right)$. There is, in particular, a flux of $p_{x}$ in the direction $\hat{z}$, namely $P_{x z}$ does not vanish.

To evaluate $\left(\mathbb{P}_{\mathrm{K}}\right)_{x z}=m \int C_{x} C_{z} f d^{3} C$ we will make several simplifying assumptions. Consider the flux accross an imaginary surface $\mathcal{S}$ at $z=$ constant and assume that a particle that crosses $\mathcal{S}$ with its velocity forming an angle $\alpha$ with the normal had its last collision at a distance $\ell$ from the 

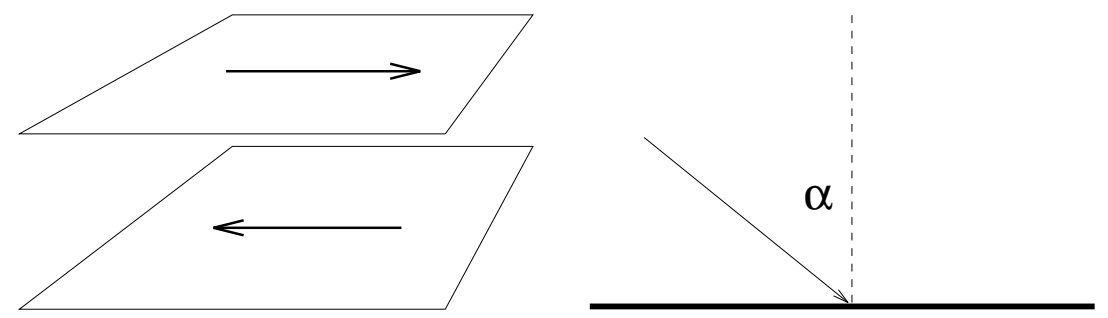

Fig. 5. If the top and bottom walls of a gas container are moved in opposite directions a strain rate $\partial v_{x} / \partial z$ is established which triggers a momentum flux. At right a particle crossing an imaginary horizontal surface with a velocity forming an angle $\alpha$ with the normal.

crossing point, namely at height $z^{\prime}=z+\ell \cos \alpha$. If it comes from above the range for $\alpha$ is $\left(0, \frac{\pi}{2}\right)$ while if it comes from below it is $\left(\frac{\pi}{2}, \pi\right)$.

The contribution to the flux from above or below the surface is then

$$
\begin{aligned}
C_{x}\left(z^{\prime}\right) & =C_{x}(z)+\ell \cos \alpha \frac{\partial C_{x}}{\partial z} \\
& =C_{x}(z)-\ell \cos \alpha \frac{\partial v_{x}}{\partial z}
\end{aligned}
$$

and the integral to be evaluated is

$$
\begin{aligned}
\mathbb{P}_{\mathrm{K}} & =\int C_{z}\left(C_{x}(z)-\ell \cos \alpha \frac{\partial v_{x}}{\partial z}\right) f d^{3} C \\
& =-\ell \frac{\partial v_{x}}{\partial z} \int C_{z} \cos \alpha f d^{3} C \\
& =-\ell \frac{\partial v_{x}}{\partial z} \int \frac{C_{z}^{2}}{C} f d^{3} C \\
& =-\eta \frac{\partial v_{x}}{\partial z}
\end{aligned}
$$

which corresponds to Newton's law of viscosity with a shear viscosity coeffcient

$$
\eta_{3 d} \approx \frac{2}{3 \sigma^{2}} \sqrt{\frac{m k_{\mathrm{B}} T}{\pi^{3}}}
$$

This is a rough result estimated via these mean free path arguments. To get (38) the distribution was assumed to be the Maxwellian $f_{\mathrm{M}}$ given in (25).

In the same fashion one can study the case of a gas between to parallel plates at different temperature. In this case there is a regime with a heat flux vector perpendicular to the plates. Making similar simplifying assumptions the result is

$$
q_{z}=-k \frac{\partial T}{\partial z} \quad \text { Fourier's law }
$$


where the thermal conductivity $k$, in this rather crude picture, turns out to be

$$
k=c_{v} \eta
$$

and $c_{v}=3 k_{\mathrm{B}} / 2 m$.

When more subtle techniques are used to derive the transport coefficients the results are different by factors which are $\mathcal{O}(1)$. For example, the lowest order result using the Chapman-Enskog method for $d=2$ or 3 are

$$
\begin{aligned}
\eta_{d} & =\frac{2+d}{2^{d+1} \sigma^{d-1}} \sqrt{\frac{m k_{\mathrm{B}} T}{\pi}} \\
k_{d} & =\frac{d(d+2)^{2} k_{\mathrm{B}}}{2^{d+2} \sigma^{d-1}} \sqrt{\frac{k_{\mathrm{B}} T}{m \pi}}
\end{aligned}
$$

Note that both coefficients, $\eta$ and $k$, have the form of a constant times $\sqrt{T}$. This is a well known property of transport coefficients for low density gases.

\subsection{Application, Thermal Slip: I}

Let us consider a gas in a box such that two opposite walls (that we conventionally call top and bottom as there is no gravity) have different temperature: $T_{b}$ and $T_{t}$ respectively and $T_{b}>T_{t}$. If the box were infinitely wide the gas would stabilize to a homogeneous temperature profile $T(z)$. To simplify let us assume that the lateral walls have precisely that temperature profile. It will be seen that the gas spontaneously slips parallel to the lateral walls towards the hotter zone, Ibsen, Soto, Cordero (1995). Later on we will be able to quantify this movement and compare the theoretical predictions with simulational results. It will be seen that the important adimensional parameter for the effect to be noticeable is $\ell|\nabla T| / T$.

The phenomenon is basically the following. Because there is a heat flux $\mathbf{q}$ accross the gas (q parallel to $\hat{z}$ ) the distribution function $f(\mathbf{r}, \mathbf{c}, t)$ is anisotropic. The gas particles hitting a point $P$, at height $z$, of a lateral wall are coming from this anisotropic distribution $f$ and, in particular, the mass flux approaching $P$ and forming and angle $\alpha$ with the normal at $P$ (see Fig.6) has an intensity that depends on $\alpha$ in a non trivial way.

Once the particles hit the wall at $P$ they get in contact with a heat bath at temperature $T(z)$ and the velocity distribution of the out-coming flux (from the wall point $P$ ) should be nearly Maxwellian. For simplicity sake it will be assumed that this out-coming flux is exactly Maxwellian and hence isotropic.

Since we will compare the theoretical predictions with results obtained with $2 \mathrm{D}$ simulations, the derivation too is made for a $2 \mathrm{D}$ system. A rough estimate of the mass flux incoming to the wall forming an angle between $\alpha$ and $\alpha+d \alpha$ with the normal is

$$
d \mathbf{J}(\text { incoming })=m n(z) v_{\text {th }}(\alpha)\left(\begin{array}{c}
\cos \alpha \\
-\sin \alpha
\end{array}\right) d \alpha
$$



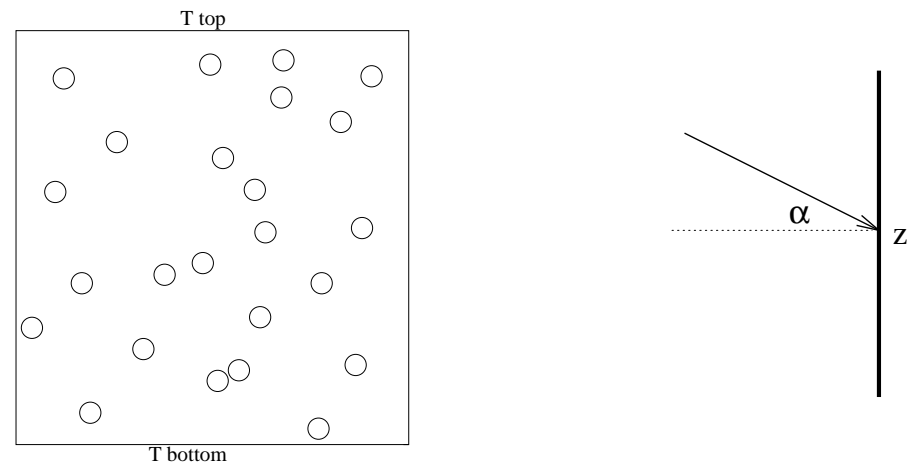

Fig. 6. At left a schematic representation of the system of particles in a square box with temperature $T_{t}$ ant the top and $T_{b}$ at the bottom. At right a particle hitting the right wall with velocity $\mathbf{c}$ forming an angle $\alpha$ with the normal to the wall.

$$
d J_{z}=-\sqrt{2 m} p \frac{\sin \alpha}{\sqrt{k_{\mathrm{B}} T(\alpha)}}
$$

where we have made use of the expression for $v_{\text {th }}$ given in table 4 and that the gas satisfies the ideal gas equation of state $p=n k_{\mathrm{B}} T$. In a gas with a temperature gradient neither the density $n$ nor the temperature $T$ are uniform but the pressure is uniform. What is implicit above is the simplifying hypothesis that all the particles that hit the wall at $P$ had their last collision at a distance $\ell$ from $P$. Since they come forming an angle $\alpha$ with the normal they come from a height $z^{\prime}=z+\ell \sin \alpha$ and at that height the temperature is what we have called $T(\alpha)$ in (43). It follows then that

$$
T(\alpha)=T(z)+\ell \sin \alpha \frac{d T}{d z}
$$

and hence

$$
\frac{1}{\sqrt{T(\alpha)}}=\frac{1}{\sqrt{T(z)}}\left(1-\frac{\ell \sin \alpha}{2 T(z)} \frac{d T}{d z}\right)
$$

The contributions to the vertical flux from $\alpha$ and $-\alpha$ do not cancel but rather give

$$
d J_{z}(\alpha)+d J_{z}(-\alpha) \propto \frac{\sqrt{m} p \sin ^{2} \alpha}{T} \frac{d T}{d z}
$$

The net mass flux is obtained integrating over $\alpha$ obtaining that it is proportional to

$$
\frac{p}{\sqrt{k_{\mathrm{B}} T}} \frac{\ell}{T} \frac{d T}{d z}
$$

Dividing this expression by the mass density $\rho=m n$ yields the velocity of the gas in contact with the lateral wall

$$
v_{z} \propto \frac{\ell}{T} \frac{d T}{d z} v_{\mathrm{th}}
$$


Noticing that $\ell \propto 1 /\left(n k_{\mathrm{B}} T\right), v_{\text {th }} \propto \sqrt{k_{\mathrm{B}} T}$ and that the Fourier law of heat conductivity $(\mathbf{q}=-k \nabla T)$ implies that $d T / d z \propto q / \sqrt{k_{\mathrm{B}} T}$ it is concluded that $v_{z} \propto q /\left(n k_{\mathrm{B}} T\right)$, namely

$$
v_{z} \propto \frac{q}{p}
$$

Analyzing the signs with care shows that the gas is pushed tangential to the wall in the direction opposite to the direction of the heat flux. This sign is derived in $\S 2.7$.

As seen from (48) this thermal slip is noticeable if the characteristic length associated to the temperature variation: $T /|\nabla T|$ is not much larger than the mean free path $\ell$ which requires either that the density be low or the temperature gradient be large or both. But there is no restriction regarding the size of the box, it can be arbitrarily larger than the mean free path.

Thermal slip is a boundary condition that has to be imposed to the hydrodynamic equations to study gases in boxes with walls having large temperature gradients parallel to them.

\subsection{Boltzmann's Equation}

Studying a statistical systems of unstructured particles satisfying a Hamiltonian dynamics it is possible to justify that the equation for the one particle distribution function $f(\mathbf{r}, \mathbf{c}, t)$, in the case of low density, is that of Boltzmann,

$$
\left(\frac{\partial}{\partial t}+\mathbf{c}_{1} \cdot \nabla_{\mathbf{r}_{1}}+\mathbf{F} \cdot \nabla_{\mathbf{c}_{1}}\right) f_{1}=\int\left(f_{1}^{\prime} f_{2}^{\prime}-f_{1} f_{2}\right) g b d b d \epsilon d^{3} c_{2}
$$

where $\mathbf{F}$ is the net instantaneous external force per unit mass acting on particle ' 1 ', $b$ is the impact parameter variable, $\epsilon$ is the azimuthal angle of the collision and

$$
\begin{aligned}
f_{1} & =f\left(\mathbf{r}, \mathbf{c}_{1}, t\right) \\
f_{2} & =f\left(\mathbf{r}, \mathbf{c}_{2}, t\right) \\
f_{1}^{\prime} & =f\left(\mathbf{r}, \mathbf{c}_{1}^{\prime}, t\right) \\
f_{2}^{\prime} & =f\left(\mathbf{r}, \mathbf{c}_{2}^{\prime}, t\right) \\
\mathbf{g} & =\mathbf{c}_{2}-\mathbf{c}_{1} \\
g & =|\mathbf{g}| \\
\mathbf{c}_{1}^{\prime} & =\mathbf{c}_{1}+\mathbf{g} \cdot \hat{k} \hat{k} \\
\mathbf{c}_{2}^{\prime} & =\mathbf{c}_{2}-\mathbf{g} \cdot \hat{k} \hat{k}
\end{aligned}
$$

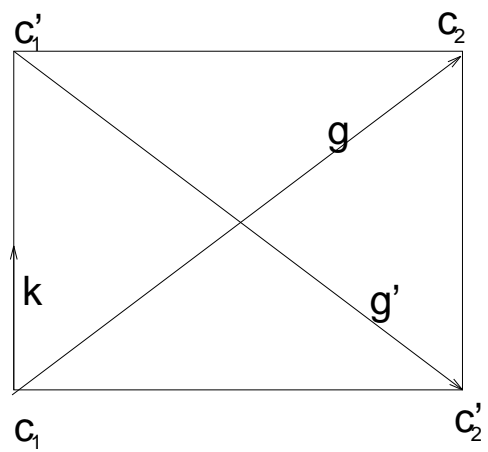

where $\hat{k}$ is the unit vector in the direction of $\mathbf{c}_{1}^{\prime}-\mathbf{c}_{1}$.

A list of the - not necessarily independent - assumptions needed to justify Boltzmann's equation are microscopic Hamiltonian dynamics, large number of particles, low density, short range interactions, inexistence of bound particles, uncorrelated particle-particle collisions, negligible spatial gradients of 
the distribution function in the atomic scale, negligible action of the external forces during the collision time.

The assumption that the collisions are uncorrelated brings up irreversibility.

\subsection{Grad's Solution}

Harold Grad in 1958 presented an original way to construct an approximate solution to Boltzmann's equation, Grad (1958). In his approximate solution he does not expand in gradients of the first five momenta of the velocity distribution (density $n$, hydrodynamical velocity $\mathbf{v}$, and temperature $T$ ) as in the Chapmann-Enskog method, but he rather uses a self-consistent approach involving higher momenta and no gradients of them. In particular he works out in detail the case when the distribution function $f$ is written in terms of $n, \mathbf{v}$ and $T$ and also the traceless and symmetric part of the pressure tensor, $p_{i j}$, and the heat flux vector $\mathbf{q}$, which in $3 \mathrm{D}$ totals 13 momenta.

Grad's approximate solution of Boltzmann's equation, $f_{\mathrm{G}}$, is obtained assuming that the distribution for a nonequilibrium system gets - in a first approximation - a corrective factor, linear in the the momenta $\mathbb{P}$ and $\mathbf{q}$, multiplying Maxwell's distribution:

$$
f_{\mathrm{G}}=(1+\mathcal{A}: \mathbf{p}+\mathcal{B} \cdot \mathbf{q}) f_{\mathrm{M}}
$$

where $\mathbf{p}$ is the symmetric and traceless part of the pressure tensor $\mathbb{P}$,

$$
P_{i j}=n k_{\mathrm{B}} T \delta_{i j}+\mathbf{p}_{i j}
$$

Grad proved that to have a solution to Boltzmann's equation consistent with the correct momenta, shown in table 3 , the specific distribution of the form (52) with $d=2$ or 3 is

$$
f_{\mathrm{G}}=\left[1+\frac{m}{p k_{\mathrm{B}} T}\left(\frac{m C^{2}}{(2+d) k_{\mathrm{B}} T}-1\right) \mathbf{C} \cdot \mathbf{q}+\frac{m}{2 p k_{\mathrm{B}} T} \mathbf{p}: \mathbf{C C}\right] f_{\mathrm{M}}
$$

\subsection{Application, Thermal Slip: II}

Let us consider again the phenomenon of thermal slip under the same conditions as before only that we consider specifically a two dimensional dilute gas of disks. Instead of making use of mean free path arguments as in $\S 2.3$ - which appeal to the intuition though render rough results - we make use of Grad's distribution (54) specialized to 2D and neglect the contribution coming from stress

$$
f_{\mathrm{G}}=\left(1+\frac{m}{p k_{\mathrm{B}} T}\left(\frac{m C^{2}}{4 k_{\mathrm{B}} T}-1\right) \mathbf{C} \cdot \mathbf{q}\right) f_{\mathrm{M}}
$$




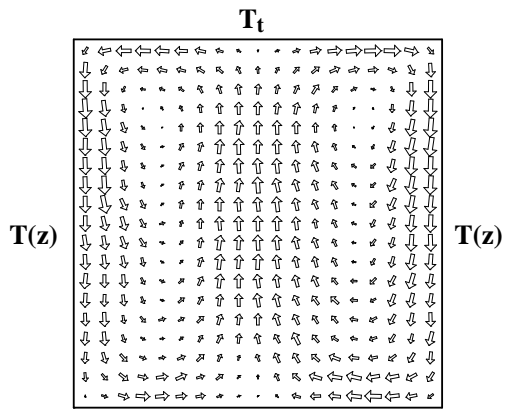

$\mathbf{T}_{\mathbf{b}}$

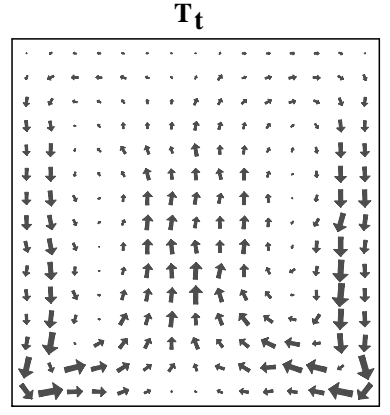

$\mathbf{T}_{\mathbf{b}}$

Fig. 7. At left the velocity field in the simulation with $N=1444, n=0.05$ and $T_{t}=0.1$. At right the thermal slip velocity field in a simulation with lateral walls made with particles with their own dynamics.

Accepting that in the bulk of the system the distribution $f_{\mathrm{G}}$ describes well the actual distribution, a natural simple assumption is that the distribution $f_{\text {rw }}$ near the right wall is

$$
f_{\mathrm{rW}}=\left\{\begin{array}{lll}
f_{\mathrm{G}} & \text { if } & C_{x}>0 \\
f_{\mathrm{M}} & \text { if } & C_{x}<0
\end{array}\right.
$$

and an entirely similar distribution near the left wall. Note that 56 defines a distribution only to lowest order as $\int f_{\mathrm{rw}} d^{2} c=n+\mathcal{O}(q)$.

With $f_{\mathrm{rw}}$ it is direct to calculate $P_{x z}=\rho\left\langle C_{x} C_{z}\right\rangle$ and the result is $\frac{q}{8} \sqrt{\frac{2 m}{\pi k_{\mathrm{B}} T}}$ which we identify, according to Newton's law, with $-\eta d v_{z} / d x$, therefore

$$
\frac{q}{8} \sqrt{\frac{2 m}{\pi k_{\mathrm{B}} T}}=-\eta \frac{d v_{z}}{d x}
$$

Assuming that the hydrodynamic velocity goes to zero exponentially with the distance to the wall, proportional to $\exp [-x / \ell]$, the right hand side above can be approximated to $-\eta v_{z} / \ell$ and (57) can be solved for $v_{z}$,

$$
\begin{aligned}
v_{z} & =-\frac{\ell}{\eta} P_{x z} \\
& =-\frac{1}{8} \frac{q}{p}
\end{aligned}
$$


We have made use of the expression for $\ell$ given in table 4 and $\eta$ from (41) with $d=2$. Equation (58) is consistent and more precise than our previous result (49). The minus sign indicates that the gas slips near the lateral walls towards the hotter regions. Fig. 7 shows at left the velocity field obtained in a simulation with 1444 particles.

In a preliminary simulation $[\mathbf{A}]$, defined below, we determined the temperature profile to impose on the lateral walls in the final simulation [B]. This was achieved running simulation $[\mathbf{A}]$ with periodic lateral boundary conditions and temperatures $T_{b}$ and $T_{t}$. The fluid stabilized in [A] with a vertical temperature profile $T(z)$. This profile was imposed in the final simulation [B] to the hard nonslip thermal lateral walls.

\begin{tabular}{|c|c|c|}
\hline & $\left|v_{z}\right|$ & $|q / 8 p|$ \\
\hline $\begin{array}{c}N=1444 \\
n=N / L^{2}=0.05 \\
T_{t}=0.1 \\
\ell \approx 7.0 \quad L \approx 170\end{array}$ & $0.015 \pm 0.002$ & $0.016 \pm 0.003$ \\
\hline $\begin{array}{c}N=8100 \\
n=N / L^{2}=0.01 \\
T_{t}=0.1\end{array}$ & $0.014 \pm 0.003$ & \\
& & \\
\hline
\end{tabular}

Table 5. Units were chosen so that the particle's diameter is $\sigma=1$, the particle's mass is $m=1$, the Boltzmann's constant is $k_{\mathrm{B}}=1$ and the temperature scale is fixed by $T_{b}=1$.

With the simulational results we measure independently the velocity $v_{z}$ of the gas in contact with the lateral wall, the pressure $p$ and the heat flux $q$. The observed results for two simulations are summarized in table 5 .

Equation (56) is the main hypothesis that lead to the quantification of this phenomenon. From the point of view of the simulation the hypothesis is concealed in the collision rule with the lateral wall: particles forget the velocity with which they hit the wall and come out with a velocity sorted out from a (isotropic) Maxwellian distribution. In case there is any doubt that the phenomenon exists at all there is a second simulation where no such hypothesis is introduced (Risso, Cordero). In this new version there is a realistic wall in the following way. The square box has periodic vertical walls, but between two relatively close parallel vertical lines we placed 420 
particles in a nearly close packing arrangement. They could not leave the space between the two lines, therefore they formed a vertical wall with its own dynamics. These two lines play the role of the effective surface cohesion between particles forming a solid. The rest of the box contained the usual dilute gas of 1444 particles. The gas particles had no intereaction with the vertical lines but they could hit the particles of the vertical wall. For both types of particles the horizontal walls had tempratures $T_{b}$ and $T_{t}$. As before we measured the velocity field of the gas and obtained the time average seen in the graph at right in Fig 7 .

\subsection{Enskog's Equation}

Enskog theory of transport arises from an extension of Boltzmann's formalism by means of introducing corrections that account for the effects of having a molecular diameter that it is no longer small compared with the mean free path. A major consequence is that the collisional mechanisms of momentum and energy transfer - which is negligible at lower densities - becomes important. In fact, collisional transfer can be as important or even more important than kinetic transfer. The contribution of the collisional transfer, in Enskog's theory, comes in when the correlations in position are introduced in the basic kinetic equation in an approximate way. In spite of these restrictions, Enskog's formalism - which was developped for the case of hard spheres - is the best systematic description that yields transport coefficients in the context of kinetic theory, Ferziger, Kaper (1972). Gass applied it to the 2D case of hard disks, Gass (1971).

Enskog's equation perfects Boltzmann's equation (50) by including effects implied by the finite size of the particles and correlations in position among them as it was already described in $\S 2.2$. If Boltzmann's equation has the form $\mathcal{D} f=J[f f]$, Enskog starts from an equation having the same left hand side as (50) but the right hand side is different,

$$
\begin{aligned}
\mathcal{D} f= & \int\left\{\chi\left(\mathbf{r}+\frac{\sigma}{2} \hat{k}, t\right) f\left(\mathbf{r}, \mathbf{c}_{1}^{\prime}, t\right) f\left(\mathbf{r}+\sigma \hat{k}, \mathbf{c}_{2}^{\prime}, t\right)\right. \\
& \left.-\chi\left(\mathbf{r}-\frac{\sigma}{2} \hat{k}, t\right) f\left(\mathbf{r}, \mathbf{c}_{1}, t\right) f\left(\mathbf{r}-\sigma \hat{k}, \mathbf{c}_{2}, t\right)\right\} \sigma^{2} \mathbf{g} \cdot \hat{k} d^{2} k d^{3} c_{2}
\end{aligned}
$$

To get Enskog's equation the right hand side is transformed expanding the functions $\chi$ and the distributions $f$ about the position $\mathbf{r}$ up to first order in the gradients. Enskog's collisional operator then has the form

$$
J_{E}=J_{0}+J_{1}+J_{2}
$$

$J_{0}$ is Boltzmann's operator $J$ multiplied by $\chi, J_{0}=\chi J$ while the other two $J_{k}$ are

$J_{1}=\sigma^{3} \int\left\{\chi \hat{k} \cdot\left(f_{1}^{\prime} \nabla_{r} f_{2}^{\prime}+f_{1} \nabla_{r} f_{2}\right)+\frac{1}{2}\left(\hat{k} \cdot \nabla_{r} \chi\right)\left(f_{1}^{\prime} f_{2}^{\prime}+f_{1} f_{2}\right)\right\} \mathbf{g} \cdot \hat{k} d^{2} k d^{3} c_{2}$ 


$$
\begin{aligned}
J_{2}= & \frac{\sigma^{3}}{2} \int\left\{\chi \hat{k} \hat{k}:\left(f_{1}^{\prime} \nabla_{r} \nabla_{r} f_{2}^{\prime}-f_{1} \nabla_{r} \nabla_{r} f_{2}\right)+\left(\hat{k} \cdot \nabla_{r} \chi\right) \hat{k} \cdot\left(f_{1}^{\prime} \nabla_{r} f_{2}^{\prime}-f_{1} \nabla_{r} f_{2}\right)+\right. \\
& \left.\frac{1}{4}\left(\hat{k} \hat{k}: \nabla_{r} \nabla_{r} \chi\right)\left(f_{1}^{\prime} f_{2}^{\prime}-f_{1} f_{2}\right)\right\} \mathbf{g} \cdot \hat{k} d^{2} k d^{3} c_{2}
\end{aligned}
$$

where the $f_{k}$ are to be understood as in (51) and Enskog's equation finally reads

$$
\mathcal{D} f=J_{0}[f f]+J_{1}[f f]+J_{2}[f f]
$$

If the system is uniform, the distribution function $f$ does not depend on position and the terms $J_{1}$ and $J_{2}$ vanish identically leading to an equation almost equal to Boltzmann's except that now the right hand side is $J_{0}$ instead of $J$.

From a conceptual point of view one should remark though that the hypothesis behind Enskog's equation are almost the same as those made explicit in $§ 2.5$ except that for Enskog's equation the size of the particles is considered and the correlations in position are somehow taken into account (sixth hypotheris in $\S 2.5$ ). The 3rd (low density) and 7th (spatial gradients of $f$ ) hypothesis are not so strongly stated in the present case. This new equation is suitable to describe a gas of hard spheres up to relatively high densities and hence it describes noble gases like Argon relatively well.

Using Chapman Enskog method of approximation, the lowest order values predicted for the viscosity and thermal conductivity for a gas of hard spheres are

$$
\eta^{(0)}=1.0160 \frac{5}{16 \sigma^{2}}\left(\frac{m k_{\mathrm{B}} T}{\pi}\right)^{\frac{1}{2}}, \quad k^{(0)}=2.522 c_{v} \eta^{(0)}
$$

In terms of these quantities Enskog's theory predicts

$$
\begin{aligned}
& \eta=\frac{1}{\chi}\left[1+\frac{4(b \rho \chi)}{5}+0.7614(b \rho \chi)^{2}\right] \eta^{(0)} \\
& k=\frac{1}{\chi}\left[1+\frac{6(b \rho \chi)}{5}+0.7574(b \rho \chi)^{2}\right] k^{(0)}
\end{aligned}
$$

where $b \rho \equiv \frac{2}{3} \pi n \sigma^{3}$ is the co-volume of the molecules and where the virial expansion for $\chi$ is

$$
\chi=1+0.6250 b \rho+0.2869(b \rho)^{2}+0.115(b \rho)^{3}+\ldots
$$

In the 2D case Enskog's theory yields the following thermal conductivity $k$ and the shear viscosity $\eta$ for the hard disk system

$$
\left.k=\frac{2.058 k_{\mathrm{B}}}{\sigma \chi} \sqrt{\frac{k_{\mathrm{B}} T}{m \pi}}\left[1+\frac{3}{2}\left(2 \rho_{\mathrm{A}} \chi\right)+0.8718\left(2 \rho_{\mathrm{A}} \chi\right)^{2}\right)\right]
$$




$$
\left.\eta=\frac{0.511}{\sigma \chi} \sqrt{\frac{m k_{\mathrm{B}} T}{\pi}}\left[1+\left(2 \rho_{\mathrm{A}} \chi\right)+0.8729\left(2 \rho_{\mathrm{A}} \chi\right)^{2}\right)\right]
$$

\subsection{Problems}

1. Write a program that defines a large set of $N$ velocity vectors $\left\{\mathbf{c}_{\mathbf{1}}, . . \mathbf{c}_{N}\right\}$. In every iteration of a loop the program takes at random two of these vectors, $\mathbf{c}_{a}$ and $\mathbf{c}_{b}$ and replaces them by vectors $\mathbf{c}_{a}^{\prime}$ and $\mathbf{c}_{b}^{\prime}$ using a reasonable collision rule with an impact parameter chosen at random. Study the evolution of the distribution of velocities starting, for example, with every c a unit vector checking that it converges to a Maxwellian rather fast. You may also study the evolution of Boltzmann's $H$ function.

2. In a similar fashion as the viscosity $\eta_{3 d}$ was derived in (38), derive the conductivity $k_{3 d}$.

3. Starting from (52) derive (54) for $d=2$ and $d=3$ requiring that the different momenta of the distribution are those of table 3 .

4. Modify the program for the 1D gas that you wrote in Prob.1.2 so that instead of rods you have point particles with the following particle-particle collision rule: $v_{1}^{\prime}=v_{2} \cos \theta+v_{1} \sin \theta$ and $v_{2}^{\prime}=v_{1} \cos \theta-v_{2} \sin \theta$ with $\theta$ chosen at random every time. The case $\theta=0$ corresponds to elastic collisions. The collision with the two extreems is specular. Notice that the energy is conserved. Measure the velocity distribution function. Is is a Maxwell distribution in the limit of a very large system?

5. Write a program for a $2 \mathrm{D}$ system circular particles of diameter $\sigma$ of two kinds: $A$ and $B$. Make each particle interacts with others of its own kind as hard disks while an $A$ with a $B$ do not repel each other but get a potential energy $-\epsilon$ while the distance $r$ between their centers is less than $\sigma$. This is a crude model of screened ions and electrons. Working with a small system check that you can get gas, liquid and solid sort of fases.

\section{Standard and high order balance equations}

\subsection{The BBGKY Hierarchy and the General Balance Equations}

None of the densities (23) or flux vectors (like $\mathbb{P}$ or $\mathbf{q}$ ) defined above can be evaluated unless the distribution function $f(\mathbf{r}, \mathbf{c}, t)$ is known. A basic problem then is to know how to obtain this distribution. The task can be formulated in many different ways. Directly from Newton's equations for every particle it is possible to derive the Liouville equation for a Hamiltonian system and from there a hierarchy of equation for distributions $f^{(n)}\left(\mathbf{r}_{1}, \mathbf{c}_{1}, \ldots, \mathbf{r}_{n}, \mathbf{c}_{n}, t\right)$ normalized to

$$
\int f^{(\nu)}\left(\mathbf{r}_{1}, \mathbf{c}_{1}, \ldots, \mathbf{r}_{\nu}, \mathbf{c}_{\nu}, t\right) d^{3} r_{1} d^{3} c_{1} . . d^{3} r_{\nu} d^{3} c_{\nu}=\frac{N !}{(N-\nu) !}
$$


satisfy

$$
\partial_{t} f^{(\nu)}+\sum_{a=1}^{\nu}\left(\mathbf{c}_{a} \cdot \nabla_{r_{a}}+\mathbf{F}_{a} \cdot \nabla_{c_{a}}\right) f^{(\nu)}=-\sum_{a=1}^{\nu} \int \mathcal{F}_{a, \nu+1} \cdot \nabla_{c_{a}} f^{(\nu+1)} d y_{\nu+1}
$$

where

$$
\mathcal{F}_{a b} \equiv-\frac{1}{m} \nabla_{r_{a}} \phi_{a b}
$$

are the interparticle forces and $f^{(\nu)} d^{3} r_{1} d^{3} c_{1} . . d^{3} r_{n} d^{3} c_{\nu}$ is the probability of having $n$ particles of the system of size $N$ about the point $\left(\mathbf{r}_{1}, \mathbf{c}_{1}, \ldots, \mathbf{r}_{\nu}, \mathbf{c}_{\nu}\right)$ regardless of where the rest of the particles are. This system of coupled equations is known as the BBGKY hierarchy and it is exact. Naturally $f^{(1)}$ is the distribution $f$ that we have been using before.

Assuming that the particles interact pairwise, making use of the first two equations of the hierarchy and without making any approximation whatsoever it is possible to find balance equations associated to quantities $\varphi$ which are additively conserved during binary particle-particle collisions

$$
\varphi_{1}+\varphi_{2}=\varphi_{1}^{\prime}+\varphi_{2}^{\prime}
$$

They are mass, momentum and energy. The exact balance equations that follow have the typical form of the equations of hydrodynamics

$$
\begin{aligned}
\frac{D \rho}{D t} & =-\rho \nabla \cdot \mathbf{v} \\
\rho \frac{D \mathbf{v}}{D t} & =\rho \mathbf{F}-\nabla \cdot \mathbb{P} \\
\rho \frac{D u}{D t} & =-\nabla \cdot \mathbf{q}-\mathbb{P}: \nabla \mathbf{v}
\end{aligned}
$$

where

$$
\frac{D}{D t} \equiv \frac{\partial}{\partial t}+\mathbf{v} \cdot \nabla
$$

is the convective derivative and the pressure tensor is the sum of a kinetic and a potential contributions

$$
\begin{aligned}
\mathbb{P} & =\mathbb{P}_{\mathrm{K}}+\mathbb{P}_{\phi} \\
\mathbb{P}_{\mathrm{K}} & =m \int \mathbf{C C} f(\mathbf{r}, \mathbf{c}, t) d^{3} c \\
\mathbb{P}_{\phi} & =-\frac{1}{2} \int \mathbf{r}_{12} \hat{r}_{12} \phi_{12}^{\prime} \int n_{2}\left(\mathbf{r}_{1}-(1-\mu) \mathbf{r}_{12}, \mathbf{r}_{1}+\mu r_{12}, t\right) d \mu d^{3} r_{12}
\end{aligned}
$$


and $\phi_{12} \equiv \phi\left(r_{12}\right)$ is the interparticle potential that is being assumed to be central and $n_{2}$ is

$$
n_{2}\left(\mathbf{r}_{1}, \mathbf{r}_{2}, t\right) \equiv \int f^{(2)}\left(\mathbf{r}_{1}, \mathbf{c}_{1}, \mathbf{r}_{2}, \mathbf{c}_{2}, t\right) d^{3} c_{1} d^{3} c_{2}
$$

On the other hand the energy density is

$$
\begin{aligned}
u & =u_{\mathrm{K}}+u_{\phi} \\
u_{\mathrm{K}} & =\frac{1}{2} \int C^{2} f(\mathbf{r}, \mathbf{c}, t) d^{3} c \\
u_{\phi} & =\frac{1}{\rho} \int \phi\left(r_{12}\right) n_{2}\left(\mathbf{r}_{1}, \mathbf{r}_{2}, t\right) d^{3} r_{2}
\end{aligned}
$$

while the contributions to the heat flux vector are

$$
\begin{aligned}
\mathbf{q}= & \mathbf{q}_{\mathrm{K}}+\mathbf{q}_{\phi 1}+\mathbf{q}_{\phi 2} \\
\mathbf{q}_{\mathrm{K}}= & \frac{1}{2} \rho \int C^{2} \mathbf{C} f(\mathbf{r}, \mathbf{c}, t) d^{3} c \\
\mathbf{q}_{\phi 1}= & \frac{1}{2} \int \phi\left(r_{12}\right) \mathbf{C}_{1} f^{(2)}\left(\mathbf{r}, \mathbf{c}_{1}, \mathbf{r}_{2}, \mathbf{c}_{2}\right) d^{3} r_{2} d^{3} c_{1} d^{3} c_{2} \\
\mathbf{q}_{\phi 2}= & -\frac{1}{4} \int \phi^{\prime}\left(r_{12}\right) \hat{r}_{12}\left(\mathbf{C}_{1}+\mathbf{C}_{2}\right) \mathbf{r}_{12} \\
& \times\left.\int f^{(2)}\left(\mathbf{r}_{1}-(1-\mu) \mathbf{r}_{12}, \mathbf{c}_{1}, \mathbf{r}_{1}+\mu \mathbf{r}_{12}, \mathbf{c}_{2}, t\right) d \mu d^{3} r_{2} d^{3} c_{1} d^{3} c_{2}\right|_{\mathbf{r}_{1}=\mathbf{r}}
\end{aligned}
$$

The vector $\mathbf{q}_{\phi 1}$ is a contribution to the potential energy flux vector while $\mathbf{q}_{\phi 2}$ contains the derivative of the potential and therefore it is related to the interparticle forces.

As we have already mentioned, the balance equations (73) are hydrodynamic equations. They are an exact and open set of equations in the sense that they are equations for the five fields $\rho, \mathbf{v}$ and $u$ but - in this context - the quantities $\mathbb{P}$ and $\mathbf{q}$ are unknowns and we have not provided equations for them within this hydrodynamic context.

To formulate hydrodynamics it is necessary to have expressions for $u$, $\mathbb{P}$ and $\mathbf{q}$ in terms of the five hydrodynamic fields $\rho, \mathbf{v}$ and $T$. The extra expressions are usually refered to as the constitutive equations.

If the particles had, for example, rotational degrees of freedom there would be an independent balance equation associated to angular momentum.

\subsection{Dilute Systems: Balance Equations and Higher Momenta}

Starting from Boltzmann's equation it is possible to derive a generic balance equation associate to any quantity $\varphi$

$$
\begin{array}{r}
\partial_{t}(n\langle\varphi\rangle)-n\left\langle\partial_{t} \varphi\right\rangle+\nabla_{r} \cdot(n\langle\varphi \mathbf{c}\rangle)-n\langle\mathbf{c} \cdot \nabla \varphi\rangle-n \mathbf{F} \cdot\left\langle\nabla_{c} \varphi\right\rangle= \\
\int \varphi\left(\mathbf{c}_{1}\right)\left(f_{1}^{\prime} f_{2}^{\prime}-f_{1} f_{2}\right) \sigma^{2} \mathbf{g} \cdot \hat{k} d^{2} k d^{3} c_{1} d^{3} c_{2}
\end{array}
$$


In particular if $\varphi$ is a quantity additively conserved in collisions then it is possible to prove that the right hand side of (79) vanishes identically and the resulting balance equations only contain kinetic contributions. They are

$$
\begin{aligned}
\frac{D \rho}{D t} & =-\rho \nabla \cdot \mathbf{v} \\
\rho \frac{D \mathbf{v}}{D t} & =\rho \mathbf{F}-\nabla \cdot \mathbb{P}_{\mathrm{K}} \\
\rho \frac{D u_{\mathrm{K}}}{D t} & =-\nabla \cdot \mathbf{q}_{\mathrm{K}}-\mathbb{P}_{\mathrm{K}}: \nabla \mathbf{v}
\end{aligned}
$$

But (79) can be used with any quantity. We have seen that the momenta that follow the first five ones (namely $n(\mathbf{r}, t), \mathbf{v}(\mathbf{r}, t)$ and $T(\mathbf{r}, t))$ of $f(\mathbf{r}, \mathbf{c}, t)$ namely $\mathbb{P}$ and $\mathbf{q}$ play a significant role.

The choices $\varphi=m\left(C_{i} C_{j}-\frac{1}{3} C^{2} \delta_{i j}\right)$ and $\varphi=m C^{2} \mathbf{C}$ yield balance equations for $\mathbb{P}$ and $\mathbf{q}$ respectively. If we further assume that the distribution function is Grad's distribution $f_{\mathrm{G}}$ then the balance equations have explicit forms shown below. These balance equations for the case $d=3$ are found in Grad's article Grad (1958), equations (28.19) and (28.20). We have derived them for $d=2$ in the hard disk case. They can be condensed for $d=2$ or 3 as:

$$
\begin{gathered}
\frac{\partial p_{i j}}{\partial t}+\frac{\partial}{\partial x_{k}}\left(v_{k} p_{i j}\right)+\frac{2}{d+2}\left(\frac{\partial q_{i}}{\partial x_{j}}+\frac{\partial q_{j}}{\partial x_{i}}-\frac{2}{d} \delta_{i j} \frac{\partial q_{k}}{\partial x_{k}}\right)+ \\
p_{r j} \frac{\partial v_{i}}{\partial x_{r}}+p_{r i} \frac{\partial v_{j}}{\partial x_{r}}-\frac{2}{d} \delta_{i j} p_{r s} \frac{\partial v_{s}}{\partial x_{r}}+ \\
p\left(\frac{\partial v_{i}}{\partial x_{j}}+\frac{\partial v_{j}}{\partial x_{i}}-\frac{2}{d} \delta_{i j} \frac{\partial v_{r}}{\partial x_{r}}\right)+\frac{1}{\tau} p_{i j}=0 \\
\frac{\partial q_{k}}{\partial t}+\frac{\partial}{\partial x_{r}}\left(v_{r} q_{k}\right)+\frac{d+4}{d+2} \frac{\partial v_{k}}{\partial x_{r}} q_{r}+\frac{2}{d+2} \frac{\partial v_{r}}{\partial x_{k}} v_{r}+ \\
\frac{2}{d+2} \frac{\partial v_{r}}{\partial x_{r}} q_{k}+\frac{k_{\mathrm{B}} T}{m} \frac{\partial p_{k r}}{\partial x_{r}}+\frac{d+4}{2 m} p_{k r} \frac{\partial\left(k_{\mathrm{B}} T\right)}{\partial x_{r}}-\frac{p_{k r}}{\rho} \frac{\partial P_{r s}}{\partial x_{s}}+ \\
\frac{d+2}{2 m} p \frac{\partial\left(k_{\mathrm{B}} T\right)}{\partial x_{k}}+\frac{d-1}{d \tau} q_{k}=0
\end{gathered}
$$

where $\tau$ is

$$
\tau=\frac{2+d}{2^{d+1}} \frac{1}{\sigma^{d-1} p} \sqrt{\frac{m k_{\mathrm{B}} T}{\pi}}
$$

As Grad has pointed out, in the absense of gradients the above equations are $\tau \partial p_{i j} / \partial t+p_{i j}=0$ and $\frac{d}{d-1} \tau \partial q_{k} / \partial t+q_{k}=0$ clearly indicating that $\tau$ is a relaxation time. But $\tau$ is proportional to the mean free flight time, $\ell / v_{\mathrm{th}}$. 
As an illustration of their use consider the first equation, neglect time variations and all gradients except for the velocity gradient. It follows that

$$
p_{i j}=-\eta_{d}\left(\frac{\partial v_{i}}{\partial x_{j}}+\frac{\partial v_{j}}{\partial x_{i}}-\frac{2}{d} \frac{\partial v_{r}}{\partial x_{r}} \delta_{i j}\right)
$$

where the shear viscosity $\eta_{d}$ for hard spheres in dimension $d$ turns out to be $\tau p$ which simplifies to (41). Similarly, from (82) the thermal conductivity for hard spheres in dimension $d$ is $d(d+2) k_{\mathrm{B}} T p \tau /(2(d-1) m)$ which is equivalent to (42).

The balance equations (81) and (82) written above can take the place of the necessary constitutive equations to have a complete hydrodynamic picture, but they really are dynamic equations. And contrary to the usual relation for these higher momenta, they do not relate linearly $\mathbb{P}$ and $\mathbf{q}$ with the gradients of $\mathbf{v}$ and $T$.

Further on we will need the nonlinear nature of these higher balance equations to be able to explain some of our observations.

\section{Thermal Conductivity}

\subsection{Introduction}

In the following we are going to carefully study the behavior of the conductivity $k$ in the absence of strain. The conductivity

$$
k(\rho, T)=k_{0}(\rho) \sqrt{T}
$$

of a two dimensional system of $N$ hard disks enclosed in a rectanguler $L_{X} \times$ $L_{Z}$ box, by means of molecular dynamics when the system has vanishing hydrodynamic velocity field (a pure conductive regime). Because the system of hard particles has no intrinsic energy, the temperature scale can only be $\sqrt{T}$ if the Fourier law is applicable.

The transport coefficients of a system of hard disks are necessary also to analyze in depth simulational results such as, for example, Bénard's convection with hard-disk systems as in Mareschall et al, Rapaport (1988), Risso, Cordero (1992).

During our simulations we can measure separately the collisional from the kinetic contributions. Determining the conductivity in this way, however, is lengthy and noisy. We have prefered to measure the net heat flux $q_{Z}$ accross the system as it would be done in real expriments and define an effective conductivity $k_{\text {eff }}$ which is the result of the conductivities that the layers of the stratified system have.

XX Tal vez los jumps debieran estar antes, tratados en detalle, todos juntos.

An important technical detail comes from a known temperature jump that occurs at the walls when the system is subjected to a temperature difference 
$\Delta T$. Because of this jump it is necessary to correct the temperatures at the walls by defining a new difference $\Delta T^{\star}$.

The system. The basic parameters that define the system are: the number $N$ of hard disks; their mass $m$, diameter $\sigma$, the size $L_{X} \times L_{Z}$ of the box, the acceleration of gravity $g$, the temperature at the bottom and top walls $T_{b}$ and $T_{t}=T_{b}-\Delta T$. It will be helpfull to use the reduced temperature difference

$$
C_{t} \equiv \frac{\Delta T}{T_{b}}
$$

Due to the important compressibility of this gas a significant density gradient would develop had we not added an accelaration of gravity $g$ in the direction of the temperature gradient.

A note of warning: having a temperature gradient and acceleration of gravity we could trigger free thermal convection. In Sec. 5 we analise this phenomenon. In the mean time it suffices to know that the simulations where performed far from the convective zone in parameter space.

The disks have elastic collisions among themselves, the vertical walls are perfectly elastic (adiabatic), while the upper and lower walls simulate contact with heat baths at the temperatures $T_{b}$ and $T_{t}$. When a particle hits a horizontal wall the tangential component of the velocity is conserved while the normal component is sorted from a Maxwellian distribution associated with the temperature of that wall.

\subsection{Heat Flux and the Effective Conductivity}

Since the Fourier law of heat conduction states that $\mathbf{q}=-k \nabla T$ we define an effective simulational bulk conductivity by

$$
k_{\text {sim }}=\frac{q L_{Z}}{\Delta T}
$$

Since the system has an important temperature gradient we directly compare the effective conductivity obtained by means of the simulations using (87) with the effective conductivity $k_{\text {theo }}$ that stems from Enskog's theory that we explain in the following paragraph.

Since $k$ depends on the local values of the density and temperature (see $(67))$ we define, in a striaghtforward manner, a theoretical conductivity $k_{\text {theo }}$ for the system as the net effect of the conductivity of all the infinitesimal horizontal slices $i$, of width $\delta$, in which the system can be thought to be devided

$$
k_{\text {theo }}=\left(\frac{1}{N_{\text {slices }}} \sum_{i=1}^{N_{\text {slices }}} k_{i}^{-1}\right)^{-1}
$$

The limit $\delta \rightarrow 0$ is understood. For our purposes, however, we have used (88) with slices of finite width to evaluate each $k_{i}\left(\rho_{\mathrm{A}}, T\right)$ from (67) and used 
the observed values of $\rho_{\mathrm{A}}$ and $T$ in each slice. The logic behind (88) is the same one as used to obtain the effective resistance $(r=1 / k)$ of a system of resistors (the horizontal slices with resistance $1 / k_{i}$ ) connected in series.

\subsection{Simulational Conditions}

To run these simulations the set of possible control parameters is rather large: $g, \Delta T$, the bulk area density $\bar{\rho}_{\mathrm{A}}, N$ and the aspect ratio $\lambda$. As we have already mentioned, the condition to have a flatter density profile is $F r \approx H\left(\rho_{\mathrm{A}}\right)$. In our simulations we took $N$ ranging from 100 to $8100, \lambda=1$ and in most simulations $\bar{\rho}_{\mathrm{A}}=0.2$ and $N=1521$ while $F r$ and $C_{t}$ were used as control parameters.

To make these observations a $15 \times 15$ standard observation mesh of cells used. To determine the length of the simulations we considered the thermal difussion time $t_{\text {diff }} \sim L_{Z}^{2} / \kappa$ (where $\kappa$ is the thermal difussivity) measured in number of collisions. Working all the details Risso, Cordero (1996) it turns out that there are about $8 \rho_{\mathrm{A}} N$ collisions per disk in one diffusion time. Typically we used a relaxation time of about $10 t_{\text {diff }}$ which, for the case of $N=1521$ and a density of $\bar{\rho}_{\mathrm{A}}=0.20$, means 24000 disk-disk collisions per disk. After relaxing the system measurements were carried out during the last 10 to 40 diffusion times.

It was checked that the equation of state (3) is satisfied at equilibrium even for large values of $g, F r$ in the range $0.3-1.7$ with $C_{t}$ up to its maximum value $C_{t}-1$. This is illustrated in the Fig. 8

The wall temperature jump off equilibrium. Once a conductive regime is established, the measured temperature difference in the bulk is smaller than the externally imposed difference $\Delta T$. This is a well known effect: Tenenbaum, Ciccotti (1982), Trozzi, Ciccotti (1984), due to a temperature discontinuity at the walls. A similar effect is observed in real, dilute systems, Hirschfelder, Cursti, Bird (1954). This temperature discontinuity is related to the collision rate and the temperature difference. In fact, the discontinuity of $T(\mathbf{r})$ near hard walls is an effect of the stochastic thermalization at the walls. A simple derivation of it is the following. The relation between the externally imposed temperature difference and the measured temperature difference can be estimated assuming local thermodynamic equilibrium and taking into consideration the rate of energy exchange in collisions with the thermalizing walls. Calling $T_{b}^{\star}$ and $T_{t}^{\star}$ the temperatures near the lower and upper walls inside the fluid, the flux of energy across the walls can be written as $q_{b}=\frac{3}{2} \Gamma_{b} k_{\mathrm{B}}\left(T_{b}-T_{b}^{\star}\right)$ and $q_{t}=\frac{3}{2} \Gamma_{t} k_{\mathrm{B}}\left(T_{t}-T_{t}^{\star}\right)$, where $\Gamma_{j}(j=b, t)$ are the disk-wall collission rates at the two thermalizing walls (XX explanation

needed). The factor $\frac{3}{2}$ comes from the velocity distribution of the particles hitting the wall. In the stationary regime it is necessary, from Fourier's law, to 


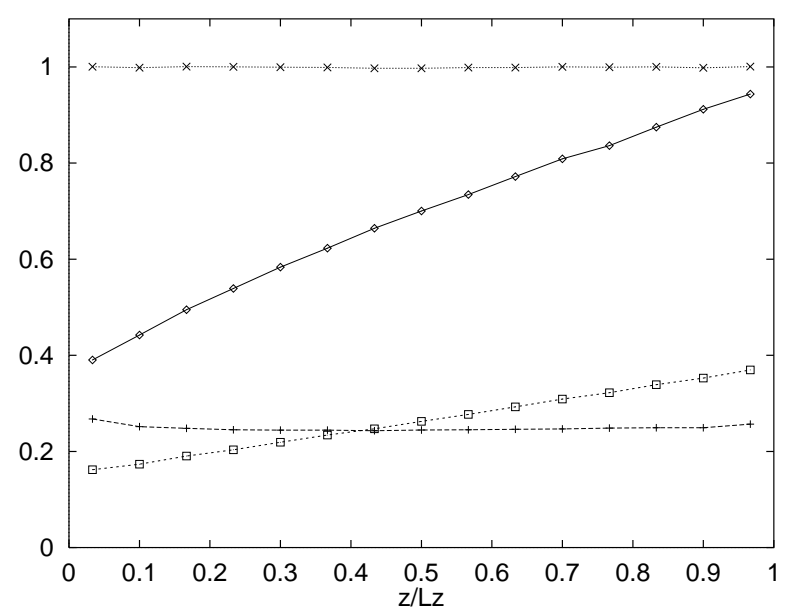

Fig. 8. Profiles of pressure $(\square)$, density $(+)$ and temperature $(\diamond)$ versus the height $z$ for a system with $N=1521, \bar{\rho}_{\mathrm{A}}=0.2, \mathrm{Fr}=1.56$ and $C_{t}=0.624$. The ratio $p L_{X} L_{Z} / N H k_{\mathrm{B}} T(\times)$ is also ploted

have $q_{b}=q_{t}=k_{\text {eff }}\left(T_{b}^{\star}-T_{t}^{\star}\right) / L_{Z}$ and from this, if the imposed temperature difference is $\Delta T$, the effective difference $\Delta T^{\star}$ is

$$
\begin{aligned}
\Delta T^{\star} & =(1+\varepsilon)^{-1} T \\
\varepsilon & \equiv \frac{2}{3}\left(\frac{1}{\Gamma_{b}}-\frac{1}{\Gamma_{t}}\right) \frac{k_{\text {eff }}}{k_{\mathrm{B}} L_{Z}}
\end{aligned}
$$

Keeping the aspect ratio $\lambda$ and $\rho_{\mathrm{A}}$ fixed, $\varepsilon$ vanishes in the limit $N \rightarrow \infty$. For a system of $N=1521, \rho_{\mathrm{A}}=0.2, C_{t}=0.5$ and $L_{Z}=78 \sigma$ the previous result yields $\Delta T^{\star} / \Delta T \approx 0.85$ which is the value that we observe.

From the concept of $\Delta T^{\star}$ it is direct to define $C_{t}^{\star}$ and $F r^{\star}$ and these are the quatities to be used, for example in (87), to make comparisons with the conductivity predicted by Enskog.

\subsection{Simulations and Theoretical Prediction}

Numerically solving the Navier Stokes (NS) equations under hydrostatic conditions making use of (a) Henderson's equation of state, (b) Fourier's law, (c) the conductivity $k\left(\rho_{\mathrm{A}}(z), T(z)\right)$ taken from Enskog's theory, (d) the effective temperature values $T_{t}^{\star}, T_{b}^{\star}$ and (e) the condition that $\bar{\rho}_{\mathrm{A}} L_{Z}=\int \rho_{\mathrm{A}}(z) d z$, it is possible to find the theoretical density, temperature and pressure profiles. Comparing these profiles with those obtained from the simulation for typical values of the imposed $F r$ and $C_{t}$ the agreement is excellent. We remark that these comparisons are not made only when $\rho_{\mathrm{A}}$ is approximately uniform namely when $\mathrm{Fr}^{*} \approx H\left(\bar{\rho}_{\mathrm{A}}\right)$ - but also when the density varies significantly 
with height. When comparing our observations with theoretical results (below), we make some remarks regarding this agreement. Note that near the top and bottom walls $\left(z=0\right.$ and $\left.z=L_{Z}\right)$ the density profile is distorted by boundary effects.

The Effective Conductivity $k$. Figure 9 shows a comparison of the values of $k$ versus $C_{t}^{*}$ obtained from the simulations with $N=1521, \bar{\rho}_{\mathrm{A}}=0.2$ and $\mathrm{Fr}^{*} \approx$ 1.56 using (87) with the theoretical conductivity, (88). To get the results summarized in figure 9 we have used seven different simulations for each $C_{t}$, starting from microscopically different initial conditions but macroscopically equivalent (same density and velocity profiles). Note that because of the statistical fluctuations in the measured values of $T_{t}^{\star}$ and $T_{b}^{\star}$ the values of $C_{t}^{\star}$ show a dispersion for every externally imposed $C_{t}$. For $C_{t}^{*}<0.3$ only one simulation was made for every $C_{t}$. Note that the observed values of $k$ are systematically larger than the theoretical values.

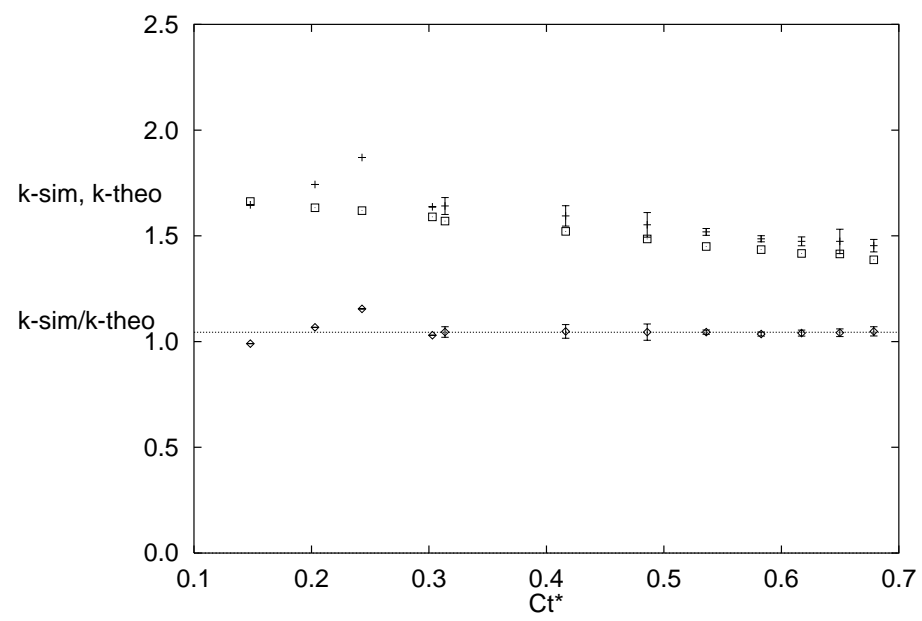

Fig. 9. Conductivities $k_{\mathrm{Sim}},(+)$ and $k_{\text {theo }},(\square)$ and the ratio $(\diamond)$ versut $C_{t}^{*}$ for a system with $N=1521, \rho_{\mathrm{A}}=0.2, \mathrm{Fr}=1.56$. The dotted line indicates the mean value 1.044 of the ratio

For a remarkably wide range of values of $C_{t}^{\star}$ the ratio $k_{\text {sim }} / k_{\text {theo }}$ is nearly constant (figure 9) in spite of the large temperature variations across the system. This can be understood from (88) - assuming that the density is about uniform (and equal to $\bar{\rho}_{\mathrm{A}}$ ) - because one can then derive that

$$
k_{\mathrm{eff}} \approx \frac{k_{0}\left(\bar{\rho}_{\mathrm{A}}\right) L_{Z}}{\int \frac{d z}{\sqrt{T(z)}}}
$$


To evaluate the ratio $k_{\text {sim }} / k_{\text {theo }}$ the same temperature profile is used in the numerator and denominator and therefore this factor cancels out. Only the ratio between the $k_{0}$ 's remains.

For the case $\bar{\rho}_{\mathrm{A}}=0.2, \mathrm{Fr}^{\star}=1.56, N=1521$ (leaving out cases for which the signal/noise ratio is too small, i.e., $\left.C_{t}^{\star}<0.5\right)$, the extrapolation from these simulational results yields $k_{\text {sim }} / k_{\text {theo }}=1.044 \pm 0.004$.

Comparison with the theoretical profiles. In the following we put forward an interpretation to explain why the observed profiles fit so well the theoretical predictions while the observed conductivity shows an indisputable difference with Enskog's prediction. In other words, how is it possible to have a discrepancy in the conductivity without having one in the profiles?

From a theoretical point of view we know that $k$ has the form (85) and any correction can be written as a corrective factor $C\left(\rho_{\mathrm{A}}\right)$ affecting the particular $k_{0}$ given in (85).

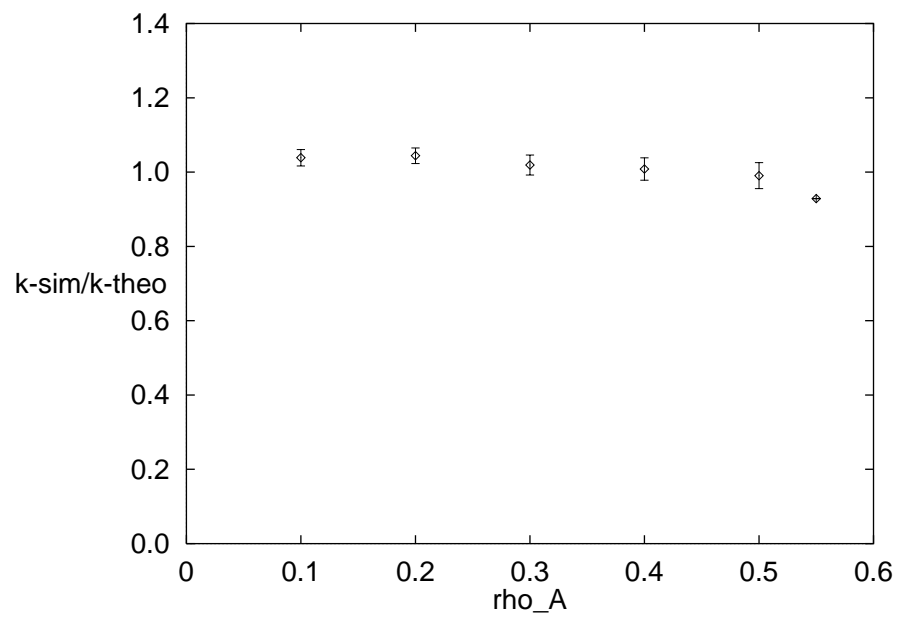

Fig. 10. Observed values for the ration $k_{\text {sim }} / k_{\text {theo }}$ for the systems with $N=1521$. The last point represents the value of this ratio reported in Mareschal, Krebs (1995)

First let us notice that the local conductivity enters the formalism only through the energy balance equation that in hydrostatics simply is $\nabla \cdot \mathbf{q}=0$, namely, $\nabla \cdot(k \nabla T)=0$. Since the only coordinate that matters is $z$ the last equation is

$$
\left(k T^{\prime}\right)^{\prime}=0 \quad \text { or } \quad \frac{T^{\prime \prime}}{T^{\prime}}=-\frac{k^{\prime}}{k}
$$

If we use (85), $k=k_{0} \sqrt{T}$, then the $T$-profile equation is

$$
\frac{T^{\prime \prime}}{T^{\prime}}+\frac{T^{\prime}}{(2 T}=\frac{k_{0}^{\prime}}{k_{0}}=\frac{1}{k_{0}} \frac{d k_{0}}{d \rho_{\mathrm{A}}} \rho_{\mathrm{A}}^{\prime}
$$


Making the change $k_{0} \rightarrow C k_{0}$ in the last expression amounts to changing

$$
\frac{1}{k_{0}} \frac{d k_{0}}{d \rho_{\mathrm{A}}} \rightarrow \frac{1}{k_{0} \frac{d k_{0}}{d \rho_{\mathrm{A}}}+\frac{1}{C}} \frac{d C}{d \rho_{\mathrm{A}}}
$$

adding a term $C^{-1} d C / d \rho_{\mathrm{A}}$ on the right hand side. From the factor $k_{0}$ in (67) one can get that $k_{0}^{-1} d k_{0} / d \rho_{\mathrm{A}}$ grows smoothly from 3.44 at $\rho_{\mathrm{A}}=0.15$ to 9.0 at $\rho_{\mathrm{A}}=0.55$ while, from the data summarized in Fig. 10, one can estimate that about $\rho_{\mathrm{A}}=0.2$ the value of $C^{-1} d C / d \rho_{\mathrm{A}}$ is about 0.013 . Namely, the observed correction to the conductivity would affect the temperature and density profiles by less than $0.3 \%$.

noindent

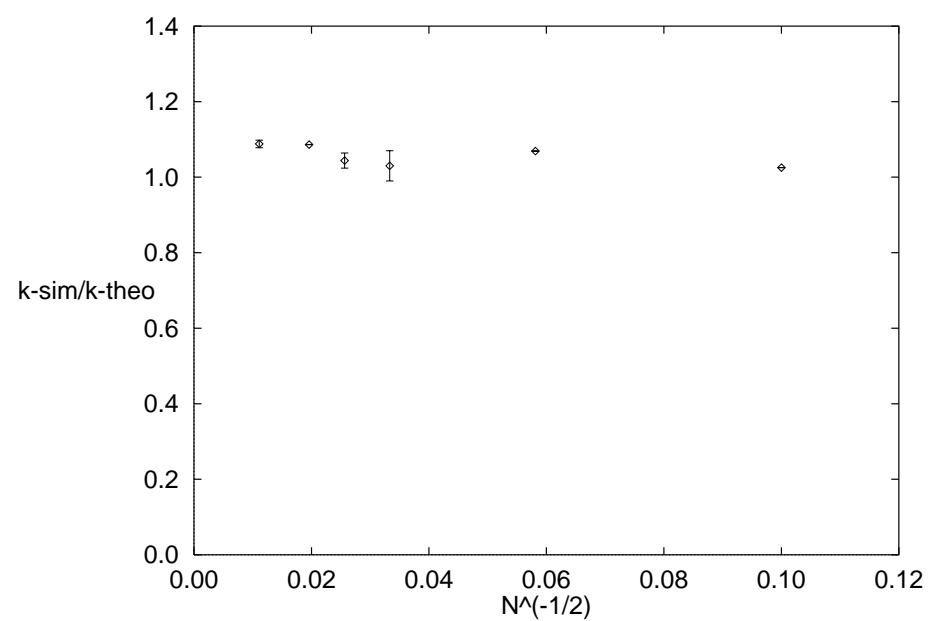

Fig. 11. The ratio between the observed theoretical values for the thermal conductivity do not show detectable size effects.

On size effects. Figure 11 shows the ratio $k_{\text {theo }} / k_{\text {sim }}$ for $N=100300,900$, 1521,2500 and 8100 . For $N=100,300$ and 2500 only one initial condition was taken while $C_{t}$ was changed (hence no error bars in these cases). For $N=8100$ only two initial conditions were considered while for $N=900$ and 1521 seven different initial conditions were considered. The cases $N=900$, 1521 and 8100 indicate that the ratio is slightly increasing with $N$ but for the excessive computational cost we disregarded the possibility of making more simulations with $N=8100$ or simulations with larger $N$. We draw no conclusions. 
Other values for the density. Though we have not made a systematic study of the conductivity as a function of the density we obtained the ratio $k_{\text {sim }} / k_{\text {theo }}$ for different densities $\left(\bar{\rho}_{\mathrm{A}}=0.1,0.2,0.3,0.4\right.$ and 0.5$)$ in the case of a system of $N=1521$ particles with $F r^{\star}=H\left(\bar{\rho}_{\mathrm{A}}\right)$. These ratios are plotted in figure 10.

We see that the most important discrepancies with Enskog's predictions seem to take place for $\rho_{\mathrm{A}} \approx 0.2$. This may seem strange since one should think that the discrepancies increase with the density. But from our results it seems that the higher order corrections that come in beyond Enskog's theory go down with the density eventually changing sign. To support this we have the above results and a recent one Mareschal, Krebs (1995) where the authors show that for a system at $\bar{\rho}_{\mathrm{A}}=0.55$ - of particles interacting with a hard (but not infinitely hard) potential - the ratio of conductivities is 0.93 . This result, seen in Fig. 10 as the last point, roughly follows the tendency of our results.

\subsection{Comments}

In the above we have assumed that the Fourier law is valid. First we have been able to obtain excellent agreement between theory and simulations regarding the density and temperature profiles when the bulk density is $\bar{\rho}_{\mathrm{A}}=$ 0.2 .

$\square \quad$ Next, we have been able to define an effective conductivity in such a way that the ratio $k_{\text {sim }} / k_{\text {theo }}$ is independent of the temperature difference for a wide range of density values $\left(\bar{\rho}_{\mathrm{A}}\right.$ from 0.1 to 0.5$)$. This independence from the temperature difference validates Fourier's law for the system and justifies extrapolating the value of the conductivity to the limiting case $\Delta T=0$ for different values of the density $\bar{\rho}_{\mathrm{A}}$.

$\square \quad$ The ratio $k_{\text {sim }} / k_{\text {theo }}$ however unequivocally differs from unity. For intermediate densities $\left(\bar{\rho}_{\mathrm{A}}\right.$ about 0.2$)$ the effective conductivity is larger than the one predicted by the theory while that difference starts to go down for larger values of $\bar{\rho}_{\mathrm{A}}$ and finally the simulational conductivity appears to be slightly smaller than the theoretical value when $\bar{\rho}_{\mathrm{A}} \approx 0.5$.

$\square \quad$ To understand why it is consistent that the profiles (item 1 above) fit so well while the conductivity does not (item 3 ), we have observed that the corrective factor $C\left(\rho_{\mathrm{A}}\right)$ multiplying Enskog's $k_{\text {theo }}$ has a sufficiently weak dependence on density - for the case $\bar{\rho}_{\mathrm{A}}=0.2$ - so that it cannot be detected in the density and temperature profiles.

\section{Compressible Free Thermal Convection}

Thermal convection in a fluid heated from below is one of the simplest cases of hydrodynamic instablity Normand, Pomea, Velarde (1977), Chandrasekhar (1961). The computational experiments of Rayleigh-Bénard (RB) convection 
by means of event driven molecular dynamics using hard disks (Mareschal, Kestemont (1987), Rapaport (1988), Puhl, Malek-Mansour, Mareschal (1989), Rapaport (1991)) has opened a new way to study dissipative structures that emerge when the system is driven off equilibrium. For the system of hard disks the compressibility effects are important in the determination of the onset of convection curve in terms of the control parameters. In fact, we have taken this as an interesting problem to be studied in detail.

The study of the linear stability problem for the compressible fluid RB convection done by Spiegel, Veronis (1976), and the nolinear stability (Gauthier, Desmarais, Ioos (1989)) as well as the numerical simulations of the NS equations (Hurl, Toomre, Massaguer (1984)) have centered their attention in fluids that obey the ideal gas equation of state but with uniform transport coefficients. In these studies, contrary to the Oberbeck-Boussinesq (OB) approximation for incompressible fluids, the density is assumed to vary widely.

In the following we present molecular dynamics results on Rayleigh-Bénard convection in $2 \mathrm{D}$ for a system of hard particles moving in a rectangular box. The system shows significant compressibility effects. Important density and temperature variations are observed when different values for the gravitational acceleration and temperature gradient are considered. We have studied the influence of these effects on the onset of convection and observed that it takes place for Rayleigh numbers significantly different to the values predicted by the Oberbeck-Boussinesq approximation. We do not limit ourselves to cases were $g$ is chosen so as to minimize the density variation with height as in Mareschal, Kestemont (1987), Rapaport (1988), Rapaport (1991), Mareschal, Kestemont (1989).

We give an empirical curve describing the onset of convection in terms of our control parameters. The results are compared with those obtained from Navier-Stokes equations when considering constant transport coefficients. There is a qualitative agreement with the theory when the work of compression is considered.

\subsection{Simulational Setting}

In principle the system has eight parameters: the number of particles $N$, the number density $n$, the aspect ratio $\lambda$, the particles diameter $\sigma$, the temperatures $T_{b}$ and $T_{t}$ at the bottom and top walls, the acceleration of gravity $g$ and the mass $m$ of the particles. The units choice $\sigma=1, m=1$ and $T_{b}=1$ leaves us with five parameters. We will see the case of a system with $N=1521$ particles in a square box (i.e., $\lambda=1$ ) and number density $n=\frac{N}{L^{2}}=0.25$. The value $\lambda=1$ guarantees that when there is convection there will be only one convective roll.

Hence we have only two control parameters that could be taken to be $g$ and $\Delta T$ but instead two adimensional numbers proportional to $g / \Delta T$ and 
$g \Delta T$ are chosen to be a Froude number and the Rayleigh number

$$
\begin{aligned}
F r & =\frac{g L}{\Delta T} \\
R a & =\frac{g \alpha n \Delta T L^{3}}{\nu \kappa} \\
& =\left(\frac{\Delta T}{T}\right)^{2} \frac{N F r}{\lambda} J(n)
\end{aligned}
$$

where $J(n)$ denpends on the number density and involves the kinematic viscosity $\nu$ and the thermal diffusivity $\kappa ; \alpha$ is the thermal expansion coefficient (7). $T$ is the mean temperature $\frac{1}{2}\left(T_{b}+T_{t}\right)$ while $\Delta T=T_{b}-T_{t}$ is the imposed temperature difference. The considered ranges for our control patameters $R a$ and $\mathrm{Fr}$ were $[100,2600]$ and $[0.5,1.7]$ respectively.

In our series of simulations the thermal diffusion time $t_{\text {diff }}$ defined in refftdiffXX corresponds to about one million total collisions or about 1300 collisions per particle ${ }^{1}$. The system was left to thermalize during $20 t_{\text {diff }}$ and measurements were taken during a $10 t_{\text {diff. }}$.

\section{$5.2 \quad$ Results}

In our simulations one could roughly say that $R a$ controls the buoyancy force while the dependence on height of the density and temperature is driven by the Frounde number $\mathrm{Fr}$.

To be able to measure the intensity of the convection in a simple way we used, as in the study of phase transitions, an order parameter which we chose to be $C$ : the integrated circulation of the velocity field, that is, the average circulation of the velocity field evaluated in concentric paths about the center of the box. This definition is appropiate because with $\lambda=1$ there can only be one stable convective roll.

The result of every simulation ( $R a$ and $F r$ fixed) was classified as nonconvective or convective according to whether the circulation $C$ did or did

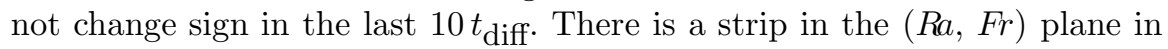
which the simulations sometimes changed sign. Such strip defines the marginal stability zone of the system which, for infinite systems and infinitely long simulations, it is a line.

Two typical plots of the evolution of $C$ in time for rather long simulations (768 million collisons) can be seen in figure 12. They correspond to $\mathrm{Fr}=1.0$ and different values of $R a$. One of these simulations shows a clear convective

\footnotetext{
${ }^{1}$ XX DINO!! En la SUBSECCI'ON SIMUlational CONDITIONS SE DIJO QUE TDIFF ERA APROX 24MIL DDC/PARTICLE CON N=1521 Y RHOA=0.2, LO QUE IMPLICA QUE TDIFF EQUiVAlE A M'AS DE 18 MILlONES DE COLISIONES. HAY UN FACTOR 20 MOLESTANDO
} 


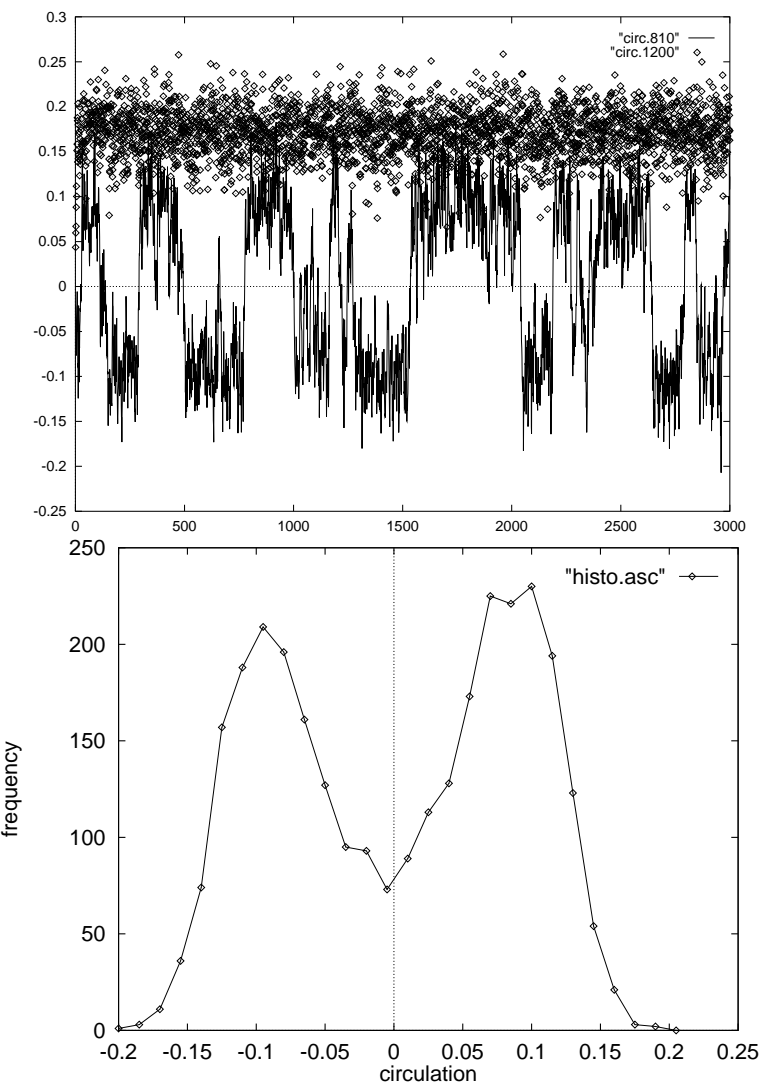

Fig. 12. At the top the evolution of the circulation $C$ during 3000 cycles of 256000 collisions each for $R a=1200$ (dots) and for $R a=810$ (continuous line). In the lower graph is the histogram for the case $R a=810$ showing that the circulation spends more time at values $\pm C_{0}$. The system has 1600 particles, $\bar{\rho}_{\mathrm{A}}=0.25, \mathrm{Fr}=1.0$.

regime $(R a=1200)$ while the other one $(R a=810)$ shows several changes of sign in its circulation. Still, in the last case the system has a value of $C$ fluctuating about a nonzero value. As we shall see, this situation is slightly above the transition from the nonconvective to convective zone.

Figure 12 also shows the histogram for the values taken by $C$. In the $R a=1200$ case the curve is a gaussian like curve about a non zero value while in the second case it is a double peaked curve with maxima about values $\pm C$.

In the OB approximation with stress free boundary conditions the critical Rayleigh number, when the aspect ratio is $\lambda=1$, is $R a_{C} \sim 780$ while in our simulations we observe that convection begins to develop for $R a$ below 700 when $\mathrm{Fr}$ is smaller that 1.0 and above 700 otherwise. Let us determine what 
is the critical valur $R a_{C}$ when $F r=1.0$.

Experimental measurements (Berge et al) and theoretical analysis (Normand, Pomea, Velarde (1977), Chandrasekhar (1961), Gorkov (1957), Malkus, Veronis (1958)) show that the square of the hydrodynamic velocity increases linearly with $R a$ near the onset of convection, $v^{2}=\frac{R a-R a_{C}}{R a_{C}} v_{0}^{2}$ therefore in figure $\mathbf{X X X b}$ we have plotted $C^{2}$ and the time fluctuations (r.m.s) of $C$ against $R a$ for simulations with $F r=1.0$. Each point in the figure corresponds to an average over a set of three simulations that differed only in their initial conditions. Each simulation lasted about 20 million collisions and the figure shows the average over the last 10 million collisions.

Assuming that after the onset of convection $C^{2}$ grows linearly with $R a$ a linear regression (the straight line in the figure) is used to determine $R a_{C}$ from the simulational points. The result is

$$
R a_{C}=712 \pm 56
$$

As we have mentioned before, one should expect that the fluctuations of the order parameter have a peak at the transition as in fact it is observed. Physically this means that near the onset of convection there is plenty of circulation but it often changes sign, hence giving a negligible average with strong fluctuations. 


\section{Delute Gas Strain Flow}

In the present paper the attention is placed on a detail description of a bidimensional Couette flow and we shall see that Grad's formalism gives the tool for a successful description. Our system will be a 2D fluid of hard disks. Thoughout this section we have set $k_{\mathrm{B}}=1$.

Several decades ago Green and Kubo introduced a method (GK method from now on) for the calculation of transport coefficients as time integrals of time correlation functions of certain microscopic currents Green (1952), Kubo (1957). With the advent of computational physics it became possible to apply the GK method to obtain estimates of the transport coefficients for particular interaction laws. These calculations led not only to the discovery of the long-time tails of the GK time correlation functions and the divergence of the GK transport coefficients in $2 D$ but also, for the 3D case to numerous specific results for self-diffusion, mutual diffusion, bulk and shear viscosity, and thermal conductivity of model gases and liquids, forming an important basis for the understanding of both the theory and phenomenology of transport.

The divergence of the 2D transport coefficients has been widely accepted (Ernst, Cichocki, Dorfman, Sharma, van Beijern (1978)) and they are expected to diverge because the corresponding correlation integrals are believed to decay slowly in $2 \mathrm{D}$, as $\mathcal{O}\left(\frac{1}{t}\right)$. On the other hand, recent high-precision simulations as in Hoover, Posch (1995), Posch, Hoover, Kum (1995), Hansen, Evans (1995), Risso, Cordero (1996) for short range steep repulsive potential (hard disks in Risso, Cordero (1996)) produced size-independent, though rate dependent transport coefficients fairly close to the predictions of Gass (1971) from the 2D Enskog theory, namely, no divergence is detected. The reproducible finite nature of viscosity in Hoover, Posch (1995) and thermal conductivity in Hansen, Evans (1995), Risso, Cordero (1996) could be made understandable if the coefficients only diverge in some unobtainable zerorange large system limit. Certainly the GK derivation of divergence fails to hold for finite systems with finite steady state nonequilibrium fluxes. One should find a bridge from finite to infinite systems, goal that is beyond our present scope.

MD simulations of Poiseuille or Couette flow in bidimensional dense fluids composed of hard disks exhibit a non Newtonian behavior (Risso (1994), Risso, Cordero (1996)) and the Fourier's law of heat conduction has also been seen to be violated in the sense that heat flux is observed in directions where there is no temperature gradient. (The temperature is understood as the local average of the kinetic energy in the co-moving frame.) To be able to make a satisfactory theoretical analysis of this behavior we have (a) performed molecular dinamic simulations of a dilute gas, observing the same behavior and (b) compared the simulational results with the implications of Grad's solution of Boltzmann's equation.

In this article we report the case of laminar stationary Couette flow. The flow gets warmer and there is a heat flux from the middle of the channel 
towards the walls. The prediction's coming from Grad's distribution describe quite well our simulational results.

We will analyze the effects of strain in the case of a Couette flow. In particular we will see results from simulations that clearly indicate that the usual linear constitutive equations of hydrodynamics cannot be applied.

\subsection{The System and a First Glance to the Observations}

The system of hard disks is inside a $L \times L$ square box, with periodic boundary vertical ( $Y$ direction) walls and the collisions among particles are perfectly elastic. The collisions with the hard horizontal ( $X$ direction) walls are such that they impose a temperature $T_{0}$ on the fluid as well as a velocity $v_{0}$ at the top wall and $-v_{0}$ at the bottom wall.

To run our simulations we have used our own algorithm Marín, Risso, Cordero (1993) and the measurement rutines described in Risso (1994). In every simulation the system was relaxed for about 20 thermal diffusion times $t_{\text {diff }}$ before local time averages of the main momenta of the distribution $(n$, $\left.\mathbf{v}, T, p_{i j}, \mathbf{q}\right)$ were taken, in some cases for as long as $\left.4000 t_{\text {diff }}\right)$.

Units are chosen so that $m=1$ and $\sigma=1$ and the temperature $T_{0}$ at the horizontal walls was set to $T_{0}=1$. The origin of the $Y$ axis is placed in the middle of the channel to make more evident the symmetry about $y=0$.

The control parameters of the simulations were the number of particles, $N=2539$ or $N=7680$, the bulk number density $\bar{n}=N / L^{2}$, and the tangencial velocity $v_{0}$ of the upper and lower walls was $v_{0}=0.25,0.5,1.0,2.0,4.0$, 8.0, 16.0, $32.064 .0,128.0$.

The bulk density $\bar{n}=4 \rho_{\mathrm{A}} / \pi \sigma^{2}$ was fixed so that the fraction of area covered by the disks was $1 \%\left(\rho_{\mathrm{A}}=0.01\right)$. With this choice the nonideal corrections to the equation of state are less than $2 \%$. The number of disks was chosen so that the ratio $c=\ell / L$, where $\ell$ is the mean free path, be small enough to keep the boundary effects constrained to a small fraction of the system and guarantee that far from the walls the fluid has a hydrodynamic behaviour. It is known that for a low density gas of hard disks $\ell=\pi \sigma /\left(8 \sqrt{2} \rho_{\mathrm{A}}\right)$ and since $L=\sigma \sqrt{N / \bar{n}}$ then $N \sim \pi /\left(32 \rho_{\mathrm{A}} c^{2}\right)$. Hence, for $N=2539$ we have $c=0.062$ and for $N=7680$ it is $c=0.036$.

\subsection{Balance Equations and Boundary Conditions}

In the present article we are interested in 2D fluids therefore we use the balance equations for that case, in particular we will use (81)and (82) with $d=2$. The $p_{i j}$ components are eliminated in favor of the complete pressure tensor $P_{i j}=p_{i j}+p \delta_{i j}$ and the expressions are restricted to the stationary 2D Couette flow with translation invariance in the $X$ direction. All physical quantities are either functions of the coordinate $y$ or they are uniform. The hydrodynamic velocity field has componments $v_{x}(y)$ and $v_{y}=0$. The mass balance equation is identically satisfied. 
In the simulations we consider a channel of width $L$ ( $Y$ direction) and periodic in the $X$ direction, with periodicity lenght $L$. The only parameters which in principle define each numerical experiment are the number $N$ of particles, the temperature $T_{0}$ and velocity $v_{0}$ and the global number density $\bar{n}=N / L^{2}$. The $y$ coordinate varies from $-L / 2$ to $L / 2$.

One has to bare in mind though that, for finite systems as ours, there are velocity and temperature jumps which cannot be neglected implying that the limit of $T(y)$ and $v_{x}(y)$ as $y \rightarrow \pm L / 2$ do not give exactly the values externally imposed. In the following We use the names $T_{0}$ and $v_{0}$ for the corrected values. Still these are the parameters that in principle should be used as our control parameters.

To fit our simulational results to the above expressions we have made the following considerations. (A) Grad's solution cannot be expected to be valid near the borders $(y= \pm L / 2)$, where the interaction with the walls plays an important distorting role particularly at low densities. (B) Assuming that Grad's solution gives the correct behavior of the system in the bulk, our expressions should reproduce the simulational results using the corrected values for $v_{0}$ and $T_{0}$.

From the two momentum balance equations (73) it follows that both $P_{x y}$ and $P_{y y}$ are uniform. From the balance equations for $P_{x x}$ and $P_{y y},(81)$ it follows that

$$
\begin{aligned}
p(y) & =P_{y y}-\frac{3}{2} \gamma(y) P_{x y} \\
P_{x x}(y) & =P_{y y}-3 \gamma(y) P_{x y}
\end{aligned}
$$

where we have introduce $\gamma$ as an adimensional measure of the strain at height $y$

$$
\gamma(y)=\tau(y) v_{x}(y)^{\prime}
$$

The rest of the balance equations yield

$$
\begin{aligned}
\frac{\tau}{2} q_{x}^{\prime} & =-P_{x y}-\gamma P_{y y} \\
q_{x}+3 \gamma q_{y} & =-\frac{6 \tau}{m} P_{x y} T^{\prime} \\
\gamma q_{x}+q_{y} & =-\frac{3 \tau}{m} P_{x y} T \gamma^{\prime}-\frac{\tau}{m}\left(4 P_{y y}+3 \gamma P_{x y}\right) T^{\prime} \\
\tau q_{y}^{\prime} & =-\gamma P_{x y}
\end{aligned}
$$

The primes indicate derivatives with respect to $y$. The above system of equation have as unknowns the fields $\gamma, T, q_{x}, q_{y}$. The boundary conditions in principle are

$$
\begin{aligned}
T( \pm L / 2) & =T_{0} \\
q_{x}(0) & =0 \\
q_{y}(0) & =0
\end{aligned}
$$


plus two integral conditions expressing that we know the global density $\bar{n}$ and the velocities $\pm v_{0}$ at the borders

$$
\begin{aligned}
\int \frac{p(y)}{T(y)} d y & =\frac{N}{L} \\
\int_{-L / 2}^{L / 2} v_{x, y} d y & =2 v_{0}
\end{aligned}
$$

In the first integral expression we have made use of the ideal gas equation of state, $p=n T$ (we are using $=1$ ), in the last expression the integrand is $\gamma(y) / \tau(y)$.

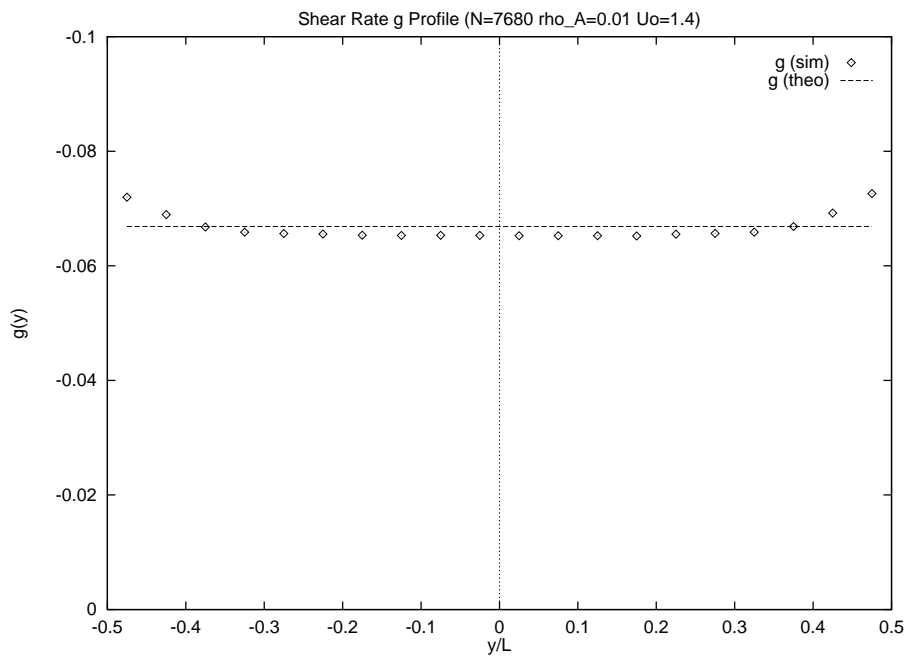

Fig. 13. The diamonds represent the observed values of $\gamma$ versus the coordinate $y / L$ for a system of $N=7680$ particles, area density $\rho_{\mathrm{A}}=0.01$ and imposed lateral velocity $v_{0}=1.4$. The straight line is the theoretical value. The discrepancy away from the borders is about XXX

\subsection{Expansions}

At first glance one cannot hope to find an analytical solution to the above system of differential equations therefore we expand in terms of a small adimensional parameter $\varepsilon$ defined in such a way that it would coincide with $\gamma$ if the strain were small and uniform,

$$
\begin{aligned}
\varepsilon & =\frac{v_{0}}{2} \sqrt{\frac{m}{\rho_{\mathrm{A}} T_{0} N}} \\
\gamma(y) & =\varepsilon+\varepsilon^{3} \eta_{3}(y)+\varepsilon^{5} \eta_{5}(y)
\end{aligned}
$$


There is a symmetry related to inverting the sign of $v_{0}$ (or $\gamma$ or $\varepsilon$ ). It is easy to see that $T, q_{y}$ and $P_{y y}$ have to be even in $\varepsilon$ while $q_{x}$ and $P_{x y}$ have to be odd functions of $\varepsilon$.

Since $q_{y}$ vanishes for zero strain its expansion begins with a term $\mathcal{O}\left(\varepsilon^{2}\right)$. Similarly, since $T^{\prime}$ vanishes for zero strain then $T=T_{0}+\mathcal{O}\left(\varepsilon^{2}\right)$. From this and (100) it follows that $q_{x}=\mathcal{O}\left(\varepsilon^{3}\right)$. Finally since for zero strain $P_{y y}$ coincides with the hydrostatic pressure and the pressure is $n T$ then we set $P_{y y}=$ $n T_{0}+\mathcal{O}\left(\varepsilon^{2}\right)$.

With all the above considerations we have solved the system of equations and their boundary conditions in a consistent way using expansions up to $\varepsilon^{6}$. All the algebraic manipulations were done using the symbolic language MAPLE. To our surprise the coefficients $\eta_{k}$ that appear in the expansion of $\gamma$ turn out to be independent of $y$ as if the strain in the bulk of the system were uniform. We conclude then that, within the theoretical picture constructed from Grad's 2D eight momentum distribution function, it is reasonable to assume that the strain $\gamma$ is uniform. The nonuniformity observed in Fig 13 when the externally imposed strain is high may be due to boundary effects and not to a deviation of the theory in the bulk where we believe that Grad's distribution is good.

\subsection{Closed solution when the strain is uniform}

Assuming that $\gamma$ does not depend on $y$ it is possible to integrate the system of equations and in particular derive that the following ratios do not depend on the temperature field

$$
\begin{aligned}
\xi \equiv \frac{P_{x y}}{P_{y y}} & =\frac{4+3 \gamma^{2}-\sqrt{\Delta}}{3\left(4-3 \gamma^{2}\right) \gamma} \approx-\gamma+\frac{9}{4} \gamma^{3} \\
\frac{q_{x}}{\gamma q_{y}} & =-\frac{18\left(2-\gamma^{2}\right)}{-3 \gamma^{2}+4+\sqrt{\Delta}}
\end{aligned}
$$

where $\Delta=16+120 \gamma^{2}-63 \gamma^{4}$. We observe that (111) implies that there is a heat flux current $q_{x}$ along the Couette channel.

Minor algebraic manipulation of the equations yield a temperature profile obeying

$$
T T^{\prime \prime}+\frac{1}{2} T^{\prime 2}+K=0
$$

hence

$$
\pm \sqrt{K} y=T \sqrt{\frac{T_{\max }-T}{T}}+T_{\max } \arctan \sqrt{\frac{T_{\max }-T}{T}}
$$

where

$$
K=\frac{81 \gamma^{4}-264 \gamma^{2}+16+\left(15 \gamma^{2}+4\right) \sqrt{\Delta}}{9 \gamma^{4}-24 \gamma^{2}+16} \frac{\pi \sigma^{2} \gamma^{2} P_{y y}^{2}}{2}
$$



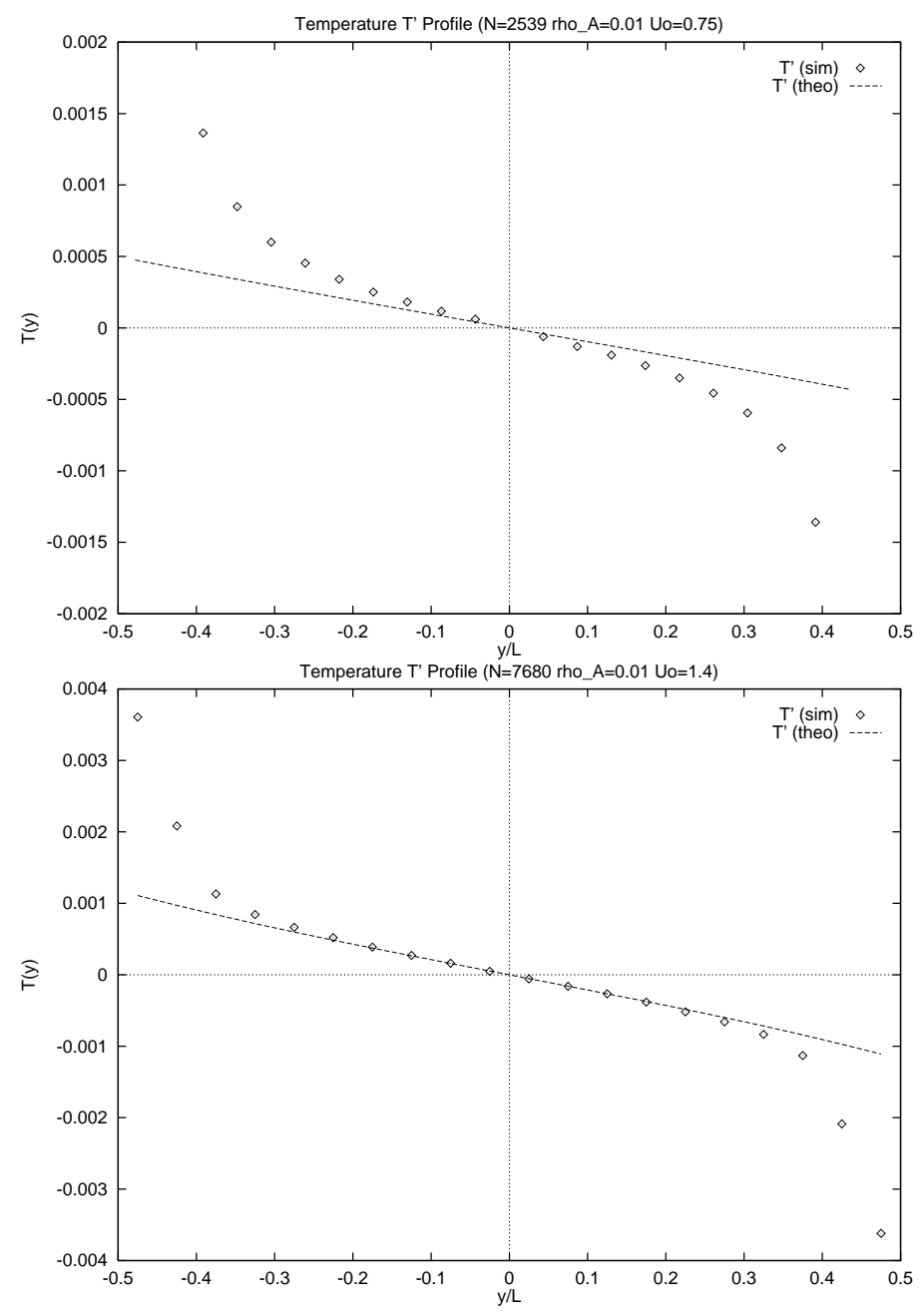

Fig. 14. The $T^{\prime}$ profile for two systems with $\rho_{\mathrm{A}}=0.01$ : $[N=2539, \gamma=-0.063]$ and $[N=7680, \gamma=-0.058]$

$K$ is positive for $0<\gamma^{2}<1 / 3$.

In practice, to integrate (112) we have imposed that $T(0)=T_{\max }$ and that $d T / d y=0$ at $y=0$. In Fig. 14 there is a comparison of the observed values of $T^{\prime}$ and the correspondig profiles obtained from the above expressions.

Combining these results it is possible to derive that the transversal heat 


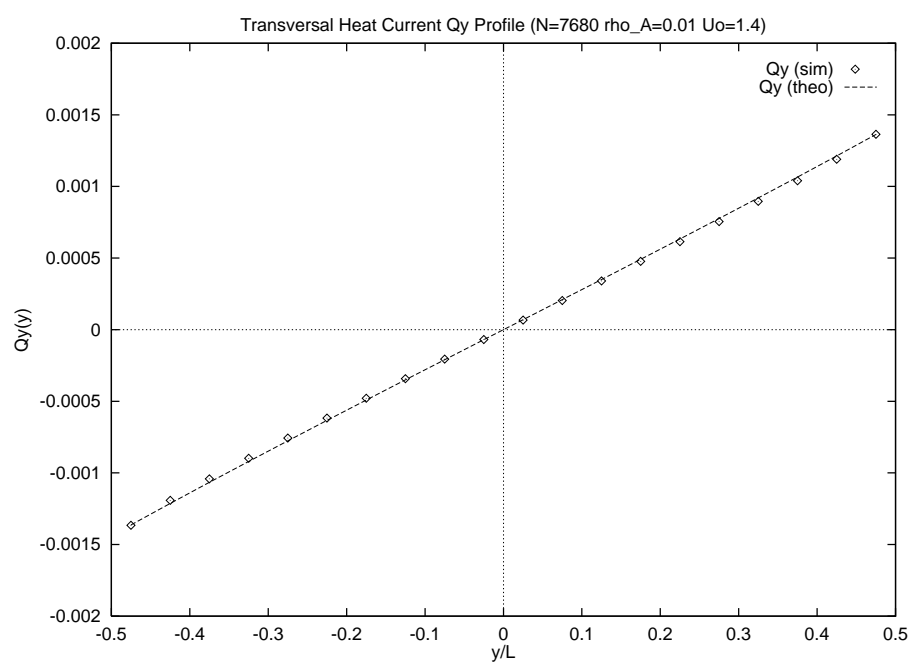

Fig. 15. Predicted and observed values of que transversal heat flux $q_{y}$ for the system with $N=7680$ and $v_{0}=1.4$

flux obeys what we could call a Fourier law

$$
q_{y}=-4 \tau P_{y y} \frac{1-\frac{3}{4} \gamma \xi}{1-3 \gamma^{2}} \frac{T^{\prime}}{m}
$$

in the sense that $q_{y}$ is proportional to $\frac{\partial T}{\partial y}$ as in (39) but the effective conductivity, $k_{\gamma}$, is seen to depend on the strain rate. In the limit of vanishing strain, $\gamma \rightarrow 0$, the conductivity is

$$
k_{0}=-\frac{4 \tau}{m} P_{y y}=\frac{2}{\sigma}, \sqrt{\frac{T}{m \pi}}
$$

which is a known first approximation to the thermal conductivity for a dilute gas of disks of diameter $\sigma$ derived using Chapmann-Enskog's method Gass (1971) or McQuarie (1976) or (42) with $d=2$.

We would like to mention that for small strain rate $\gamma$ the expression (110) can be written as

$$
P_{x y}=\frac{1}{2 \sigma} \sqrt{\frac{m T}{\pi}} v_{x}^{\prime}
$$

which is Newton's viscous flow law, see (41). But (110) gives a quite nonlinear relation between $P_{x y}$ and the strain rate.

\section{Results}

In this section we describe the way we made the observations during our simulations and then proceed to compare the simulational results with those coming from the expressions of the previous section. 


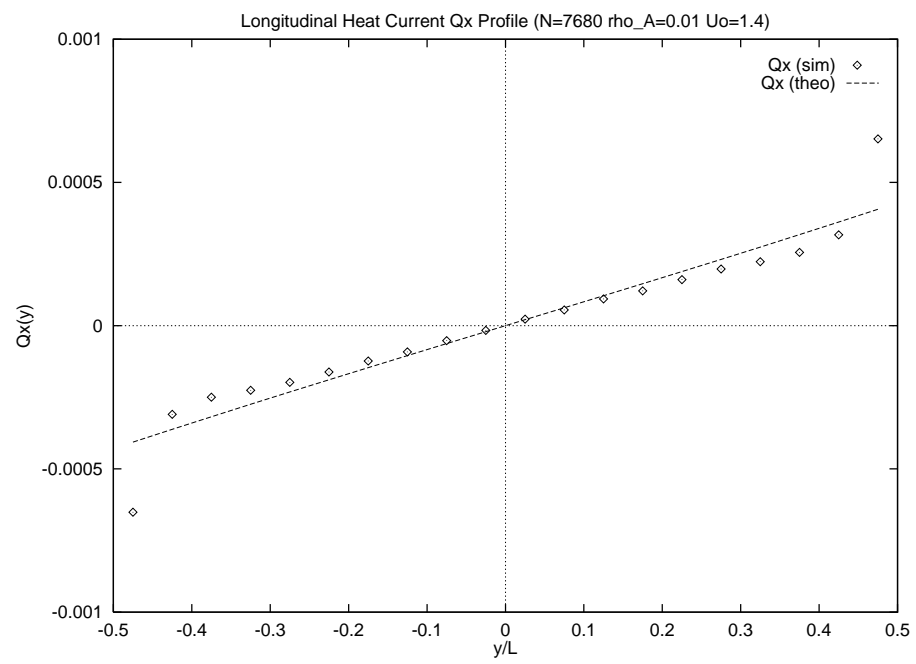

Fig. 16. Predicted and observed values of the longitudinal heat flux $q_{x}$ for the system with $N=7680$ and $v_{0}=1.4$ There is no temperature gradient in this direction.

To observe the hydrodynamic behavior of our system, the box was divided in $M_{x} \times M_{y}$ rectangular cells. In each cell the time average of the first momenta of the distribution are made. For the system with $N=7680$ particles we took $M_{x}=M_{y}=20$, which corresponds to about 19.2 disks per cell and in the case with $N=2539$ we took $M_{x}=M_{y}=23$ or about 4.8 disks per cell.

A summary of the observations. Most quantities show boundary effects. The temperature field shows isotherms parallel to the flow but the heat flux is not orthogonal to them: it bends in the direction of the mass flow. The equation of state is well satisfied all accross the fluid, incluiding the regions near the walls. Observed discrepancies with the ideal gas equation were always below 2\% and if Henderson's equation of state (4) is used then the discrepancies are below $0.1 \%$ in the case of the system with $N=7680$ particles. The component $P_{x y}$ and $P_{y y}$ of the pressure tensor show no boundary effects but $P_{x x}$ does.

Taking advantage of the translation invariance in the $X$ direction, we took horizontal averages of the observed cell-results getting in this way vertical profiles for the main momenta.

As we have already mentioned, $P_{x y}$ and $P_{y y}$ should be uniform. From the horizontal averages of $P_{x y}$ and $P_{y y}$ their values at each $y$ are obtained with errors of less than $0.6 \%$ and less than $0.07 \%$ respectively for $N=2539$ particles. For the larger system the errors are smaller. An additional vertical average over each of the previous profiles show that these two profiles are 
independent of $y$ with a variance of about $0.1 \%$ for $P_{x y}$ and of about $0.008 \%$ for $P_{y y}$ when $N=2539$ and smaller when $N=7680$.

Even though we derived that up to 6 th order in $\varepsilon$ the adimensional strain $\gamma$ is uniform, in our simulations for strains as small as $\varepsilon=0.06$ there is a wide region near the boundaries of the channel where $\gamma$ noticeable varies with $y$. But in the central region $\gamma$ is quite uniform. See Fig.13.

From this considerations we see that the theoretical framework presented in $\S 6.2-\S 6.4$ needs a reassessment because, even though the differential equations are expected to be valid in the bulk, this is not true near the boundaries. The closed expressions found in $\S 6.4$ should be expected to fit well away from the walls and the values associated to the boundary conditions $\left(T_{\max }, v_{0}\right)$ must be adjusted to make this fit.

To adjust our results we proceeded as follows:

(a) As we have seen, both $P_{x y}$ and $P_{y y}$ are independent of the coordinate $y$. This is in fact observed to be true within a small margin hence we take averages of the vertical profiles of these quantities. With these averages and (110) we have determined an effective value $\gamma^{*}$.

(b) From the observed temperature profile and the expression (113) we have used mean squares to determine the best fit for $T_{\max }$. In practice we get slightly different fits if we eliminate one value of $T$ at each extreem than if we eliminate two or three etc values. From all these values for $T_{\max }$ we obtain an extrapolated final number $T_{\max }$.

Having the value for $P_{y y}$ and $\gamma$ we have the value of the constant $K$. With it and $T_{\max }$ we get what we have called the theoretical temperature profile $T(y)$ and therefore its gradient $T^{\prime}(y)$. We compare the latter with the observed 
values in Fig. 14. We have not shown directly $T(y)$ because the agreement is not very good, the curvature of the simulational profile is larger than the theoretical one. The $T^{\prime}$ profile, however agrees quite well when the system is larger and away from the walls.

(c) Finally, from the observed values for $P_{x y}$ and $P_{y y}$, the value $\gamma^{*}$ and the theoretical profiles of $T$ and $T^{\prime}$ we obtain, using the expressions (97), (111) and (115) the theoretical profiles for $p, q_{y}$ and $q_{x}$ that are used to compare with the values directly observed for these quantities.

Figure 13 shows the theoretical values of $\gamma$ derived from (3) with the horizontal averages of $P_{x y}$ and $P_{y y}$ and the simulational values of $\gamma$ obtained from (98), using the local values of $p, v_{x}^{\prime}$ and $T$. The figure corresponds to the case $N=7680$ and $v_{0}=1.4$. Away from the walls the predicted value of $\gamma$ is $\gamma=0.0669 \pm 0.0004$ which coincides with the observed value within a $2 \%$ margin.

In Fig. 14 the finite size effects on the profile for $T^{\prime}$ is shown for two systems, both with $\gamma \approx-0.065$. The larger system shows a much better agreement and the range of the distortion near the boundaries is smaller.

Figure 15 shows the value of the transversal heat current $q_{y}$ for the case $N=7680, v_{0}=1.4$. Notice that $q_{y}$, as seen in (115) obeys a Fourier type of law with an effective conductivity that depends on the rate of strain. The agreement with the theory is excellent.

In figure 16 we plot the component of the heat flux current $q_{x}$ along the isotherms for two systems of different size, $N=2539$ and $N=7680$ with about the same strain $\gamma \approx 0.065$. It is seen that the agreement is quite good, particularly for the larger system. It seems that for $q_{x}$ the boundary effects propagate deeper into the system, but these effects go down as the size of the system is increased.

\section{Acknowledgments}

This work was supported by the Fundación Andes grant C-12971 and CNRSConicyt grant. One of us (D.R.) is also thankfull of the research grants Diprode 951005-2 and 960105-6.

\section{References}

Arroyo M.P. and Savirón J.M., J. Fluid Mech. 235325 (1992).

Alder B.J. and Wainright T.E., J. Chem. Phys. 31, 459 (1959).

Alder B.J. and Wainwright T.E., Phys. Rev. Lett. 18 988, (1967); Phys. Rev. A1 18 (1970); J. Chem. Phys. 533813 (1970).

Allen M.P. and Tildesley D.J. Computer Simulation of Liquids, Oxford Science Pub, 1989

Barker J. A. and Henderson D., What is a liquid?, Rev. Mod. Phys. 48587 (1976). 
Berge P. and Dubois M., J. Fluid Mech. 321041 (1974); P. Berge Fluctuations, Instabilities and Phase Transitions, T. Riste ed. Plenum Press, N.Y. 1975; M. Dubois and P. Berge, J. Fluid Mech. 85641 (1978).

Chandrasekhar S. , Hydrodynamic and hydromagnetic stability, Clarendon Press, Oxford, 1961.

Chapman S. and Cowling T.G. The Mathematical Theory of Nonuniform Gases, Cambridge University Press, third edition, 1970

Ciccotti G., Hoover W.G. (eds) , Molecular Dynamics Simulation of Statistical Mechanical Systems, North Holland 1986.

Ciccotti C., Frenkel D. and McDonald I.R. (eds), Simulation of Liquids and Solids, North Holland, 1987.

Cordero P., Marín M. and Risso D., Chaos, Solitons and Fractal 695 (1995)

Davis H.T., Rice S.A. and Sengers J.V. , J. Chem. Phys. 352210 (1971); Schrodt J.B., Ku J.S., and Luks K.D., J. Chem. Phys. 574589 (1972).

Ernst M.H., Cichocki B., Dorfman J.R., Sharma J. and van Beijern H. Jour. Stat. Phys. 18237 (1978)

Evans D.J., Phys. Rev. A 221988 (1980).

Evans D.J. and Morris G. P., Statistical Mechanics od Nonequilibrium Liquids, Academic Press, 1990.

Hansen D.P. and Evans D. J., Molecular Simulation 14409 (1995).

Ferziger J.H. and Kaper H.G. Mathematical Theory of Transport Processes in Gases, North-Holland 1972

Gass D.M., J Chem Phys 541898 (1971).

Gauthier S., Desmarais T. and Ioos G., Europhysics Lett. 10543 (1989); S. Gauthier, A. Gammes and G. Ioos, Europhysics Lett. 13117 (1990)

Glikman E., Kelson I., Doan N.V. and Tietze H., Journ. Comp. Phys. 12485 (1996).

Gorkov L.P., Sov. Phys. JEPT 6311 (1957).

Grad H., Principles of the Kinetic Theory of Gases in Handbuch der Physik, Vol. XII, edited by S. Flunge, Springer, (1958); Theory of Rarefied Gases in Rarefied Gas Dynamics, edited by F. Devienne, Pergamon Press, 1960.

Green M. S., J. Chem. Phys. 201281 (1952); M.S. Green, J. Chem. Phys. 22398 (1953); R. Kubo, J. Phys. Soc. (Japan) 12570 (1957).

Hansen J.-P. and McDonald I.R. Theory of Simple Liquids, Academic Press 1986, second edition reprinted in 1991.

Henderson D., Mol. Phys. 30971 (1975)

Heyes D.M., J. Phys: Condens Matt. 66409 (1994)

Hirschfelder J. O., Curtis C. F. and Bird R. B., Molecular Theory of Gases and Liquids (Wiley, New York, 1954).

Hoover Wm. G., Molecular Dynamics, Springer Verlag, Berlin, 1986.

Hoover W.G. and Posch H.A., Phys. Rev. E 51273 (1995).

Hurl N., Toomre J. and Massaguer M., Astrophysics J. 282557 (1984)

Ibsen J., Soto R. and Cordero P. Phys. Rev E 524533 (1995).

Knuth D.E., The Art of Computer Programming (Addison-Wesley, Reading, MA, 1973)

Kratky K.W. , J. Chem. Phys. 692251 (1978).

Lifshitz E.M. and Pitaevskii L.P. Physical Kinetics Pergamon Press, reprinted in 1993 
Lubachevsky B. D., Journ. Comp. Phys. 94255 (1991)

McQuarie D. A., Statistical Mechanics Harper Collins Publishers, 1976.

Maeseo M.J. and Solana R., J. Chem. Phys. 99548 (1993).

Malkus W.V.T. and Veronis G., J. Fluid Mech.4 225 (1958).

Mareschall M. and Kestemont E., Nature 329427 (1987); M. Mareschal and E. Kestemont, J. Stat. Phys. 48, 1187 (1987); A. Puhl, M. Malek-Mansour and M. Mareschal, Phys. Rev. A 40, 1999 (1989); M. Mareschal, M. Malek-Mansour, A. Puhl and E. Kestemont, Phys. Rev. Lett. 41, 2550 (1988)

Mareschal M., Kestemont E., Nature 427329 (1986).

Mareschal M., Kestemont E., J. Stat. Phys. 481187 (1987).

Mareschal M., Kestemont E., Phy Lett. 62691 (1989).

Mareschal M. and Krebs U. in Proceedings of the Eurotherm 36 Conference

Marín M., Risso D. and Cordero P. Journ. Comp. Phys. 103306 (1993).

Marín M. and Cordero P., Computer Physics Communications 92214 (1995).

Normand C., Pomeau Y. and Velarde M. , Rev. Mod. Phys. 49581 (1977).

Posch H.A. , Hoover W. G. and Kum O., Phys. Rev. E 521711 (1995).

Puhl A., Malek-Mansour M. and Mareschal M., Phys. Rev. A 401999 (1989).

Rapaport D.C., Journ. Comp. Phys. 34184 (1980).

Rapaport D. C., Phys. Rev. Lett. 602480 (1988); D. C. Rapaport, Phys. Rev. A 461971 (1992).

Rapaport D.C., Phys. Rev. Lett 437046 (1991).

Résibois P. and de Leener M. Classical Kinetic Theory Wiley 1977.

Risso D. and Cordero P., In Condensed Matter Theories, A. N. Proto and J. Aliaga, eds. (Plenum Press, New York, 1992); D. Risso and P. Cordero, In Proceedings of the IV Instability and Nonequilibrium Structures, E. Tirapegui and W. Zeller, eds. (Kluwer, Dordrecht, 1992).

Risso D., Doctoral thesis, Universidad de Chile, May 1994.

Risso D. and Cordero P., J. Stat. Phys. 821452 (1996).

D. Risso and P. Cordero in Instabilities and Nonequilibrium Structures $V$, E.

Tirapegui, W. Zeller (eds), Kluwer Acad. Publishers, 1996.

Risso D. and Cordero P. unpublished.

Spiegel E., Veronis G., Astrophysics J. 131442 (1960); E. Spiegel, Astrophysics J. 1411068 (1965); D. Gough, D. Moore, E. Spiegel and N. Weiss, Astrophysics J. 206536 (1976)

Tenenbaum A., Ciccotti G. and Gallico R., Phys. Rev. A 752778 (1982)

Trozzi C. and Ciccotti G., Phys. Rev. A 29916 (1984)

Wesfreid J., Pomeau Y., Dubois M., Normand C. and Berge P., Le Journal des Physique - Letters 39725 (1978). 